\title{
One-pot multicomponent nitro-Mannich reaction using a heterogeneous catalyst under solvent-free conditions
}

\author{
Giovanna Bosica Corresp., 1 , Ramon Zammit ${ }^{1}$ \\ ${ }^{1}$ Department of Chemistry, University of Malta, Msida, Malta \\ Corresponding Author: Giovanna Bosica \\ Email address: giovanna.bosica@um.edu.mt
}

An environmentally-friendly, one-pot multicomponent reaction of various aldehydes, amines and nitroalkanes for the synthesis of $\beta$-nitroamines is here described. Amberlyst $A$ 21 supported Cul was found to be a highly efficient novel heterogeneous catalyst for the three-component nitro-Mannich reaction between aldehydes, amines and nitroalkanes. The developed protocol is performed in a solvent-free medium to produce a variety of $\beta$ nitroamines in good to excellent yields within short reaction times. The catalyst can be easily prepared and recovered. It has been tested up to 8 times with only a minor activity loss. 


\title{
1 One-pot multicomponent nitro-Mannich reaction using a 2 heterogeneous catalyst under solvent-free conditions
}

3

4 Giovanna Bosica ${ }^{1 *}$ and Ramon Zammit ${ }^{1}$

$5 \quad{ }^{1}$ Department of Chemistry, University of Malta, Msida, Malta 6

7 Corresponding Author:

8 Giovanna Bosica ${ }^{1}$

9 Department of Chemistry, University of Malta, Msida MSD2080, Malta

Email address: giovanna.bosica@um.edu.mt

\section{Introduction} nucleophile, an enolisable carbonyl compound, has been replaced by a nitroalkane.

Figure 1

\begin{abstract}
An environmentally-friendly, one-pot multicomponent reaction of various aldehydes, amines and nitroalkanes for the synthesis of $\beta$-nitroamines is here described. Amberlyst A-21 supported CuI was found to be a highly efficient novel heterogeneous catalyst for the three-component nitroMannich reaction between aldehydes, amines and nitroalkanes. The developed protocol is performed in a solvent-free medium to produce a variety of $\beta$-nitroamines in good to excellent yields within short reaction times. The catalyst can be easily prepared and recovered. It has been tested up to 8 times with only a minor activity loss.
\end{abstract}

The synthesis of bioactive secondary metabolites through the formation of networks of carboncarbon bonds has fascinated synthetic organic chemists for entire generations [1]. The nitroMannich reaction is a Mannich-type reaction (Fig. 1), an imine-based reaction, where the original

It is a powerful $\mathrm{C}-\mathrm{C}$ bond forming reaction which involves the addition of nucleophilic nitronate species, derived from nitroalkanes, to electrophilic imines [2,3]. The resulting $\beta$-nitroamine product is considered to be a privileged building block because it possesses potential vicinal stereocentres, bearing nitro and amino functional groups with different oxidation states, providing 
31 opportunities for the selective manipulation of either in subsequent transformations $[4,5]$. $\beta$ 32 Nitroamines serve as useful intermediates to access other valuable moieties such as 1,2-diamines (via nitro reduction) [6], $\alpha$-amino carbonyls and $\alpha$-amino acids (via the Nef reaction) [7], peptides synthesis of umpolung chemistry [8] and monoamines (via reductive denitration). The nitro functional group can also form other functionalities such as hydroxylamines [9], and oximes, or nitriles [10]. Indeed several natural products and pharmaceuticals have been synthesised via an intermediate nitro-Mannich reaction including antiemetic [11], analgesics [12], antimalarial and antiinsecticidal [13], antipsychotic [14,15], antiviral [16], anticancer [17,18], anti-HIV [19], antiparasitic [20], antidepressant [21], antibacterial and antimicrobial [22], and anaesthetic [23].

Yet despite the utility, research in the nitro-Mannich reaction has not been very popular because the $\beta$-nitroamine product is often prone to retro-addition and epimerisation [24]. Moreover, the addition of nitronates to imines is not thermodynamically favoured due to the difference between the $\mathrm{pKa}$ values of nitroalkane ( $\mathrm{pKa} \sim 9$ ) and the $\beta$-nitroamine product ( $\mathrm{p} K \mathrm{a} \sim 35$ ) [2]. As a result, Lewis or Brønsted acids were suggested to activate the electrophilic imine [25-27], or organic and inorganic bases to activate the nitroalkane, to form the nitronate species [28-31]. These catalysts, however, present a number of disadvantages since big amounts are applied, certain metals used are very expensive, and only function in harsh temperature conditions, with large excess of nitroalkane and in the presence of hazardous solvents. In this regard, there has been a strategic focus to develop more environmentally friendly nitro-Mannich protocols. This has been achieved in two ways: the employment of a heterogeneous or recyclable catalyst [32-34], which can be recovered and reused, or the application of a one-pot multicomponent strategy between aldehydes or ketones, amines and nitroalkanes (Fig. 2) [35-37].

\section{Figure 2}

Heterogeneous catalysts have been shown to also catalyse like the homogeneous ones with some advantages. In fact they are non-corrosive, safely handled, porous material increasing the surface area, result in more effective encounter rates, are easily recovered from the reaction mixture without the need for reaction quenching and can be recycled and reused [38]. Following our studies for the development and application of heterogeneous catalysts in multicomponent reactions [39], herein we wish to report a simple and efficient approach to the one-pot three-component nitroMannich reaction using a recyclable heterogeneous catalyst. 


\section{Results and Discussion}

\section{Catalyst Screening}

64

Initial investigation involved employing a series of catalysts to screen the nitro-Mannich step between $N$-benzylideneaniline, $(\mathbf{5}, 1 \mathrm{mmol})$ and nitromethane, $(\mathbf{3 a}, 10 \mathrm{mmol})$ as model reaction (Fig. 3). Due to the relative ease in the formation of the intermediate imine, screening for catalyst selection was only performed on the second more challenging nitro-Mannich step, from preformed imines. Various heterogeneous catalysts or catalytic species which could be immobilised on a solid support were all employed under solvent -free conditions. Both organic and inorganic bases, and Lewis or Brønsted acids were used to potentially activate the nucleophilic nitroalkane or electrophilic imine, respectively.

Catalysts tested include species which have been previously tried in the nitro-Mannich reaction such as carbonates, bicarbonate and hydroxide inorganic salts (entries 2-6) [40-42], silica (entry 7) [43], basic alumina (entry 8) [44], and KF on alumina (entry 16) [32], as well as species which have been documented to function efficiently in similar Mannich-type reactions such as Amberlite IRA-400 Cl resin (entry 10) used to catalyse the aza-Friedel Crafts reaction [45], montmorillonite K-10 (entry 12) used to synthesise numerous Mannich bases [46], PPA-silica (entry 13) used in double Mannich condensation reactions [47] or similar $\mathrm{C}-\mathrm{C}$ bond forming reactions such as Amberlyst A-21 (entry 9) used to catalyse the nitroaldol reaction [48]. Since modest results were observed with different acidic and basic strengths, a number of bifunctional catalysts were tested. Amberlyst A-21 supported copper(I) iodide (entry 17) was previously used in the $\mathrm{A}^{3}$ - and $\mathrm{KA}^{2}$ coupling reactions, an acetylene-Mannich reaction [49,50]. Given the Lewis acid $\mathrm{Cu}^{+}$ions and basic tertiary amino-grafted polystyrene polymer, it was postulated that its activity could also be extended to the formation of $\beta$-nitroamines. Complete conversion for the synthesis of $\mathbf{4 a}$ occurred at a faster rate $(48 \mathrm{~h})$ and the product was isolated with a good yield, confirming the hypothesis. Substitution of the transition metal, (entry 19), metal counter ion, (entry 18), or metal support, (entries 20,21), resulted in a reduction of the catalytic activity or lower product yield. As a result, Amberlyst A-21 supported CuI was selected for the basis of this study.

Figure 3

\section{Reaction Optimisation}


91 In order to develop a greener approach to the nitro-Mannich reaction, efforts were made to employ 92 Amberlyst A-21 supported $\mathrm{CuI}$ in a one-pot three-component strategy reaction between benzaldehyde (1a) and aniline (2a) (the starting materials required to form $N$-benzylideneaniline, 5) together with nitromethane (3a) (Fig. 4). The multicomponent reaction was performed in a sequential manner such that the catalyst, CuI-Amberlyst A-21, 1a and 2a were first mixed to form the intermediate 5 followed by addition of $\mathbf{3 a}$ in the same pot to form the resultant $\beta$-nitroamine $\mathbf{6}$. Using the same reaction conditions as the two-step strategy, the reaction was complete after $48 \mathrm{~h}$ and the isolated yield of $\mathbf{6}$ was slightly improved, confirming the efficiency of the heterogeneous catalyst to catalyse the multicomponent reaction.

\section{Figure 4}

Efforts were then made to optimise the reaction conditions of the model multicomponent reaction. A number of factors were evaluated including catalyst loading, temperature, and molar ratio, in particular the amount of excess nitroalkane (Fig. 5). Using as little amount of catalyst as possible is essential to minimise its cost. In fact, no improvement in activity or yield was observed when increasing the catalyst loading to 10 and $20 \mathrm{~mol} \%$ (entries 2, 3) from the original $5 \mathrm{~mol} \%$ used (entry 1). However, further decreasing the catalyst amount to 2 and $1 \mathrm{~mol} \%$ (entries 4, 5) resulted in a negative effect both on the reaction time and yield. Concerning the molar ratio, the reaction is preferably performed in a stoichiometric ratio of starting material since excess reagents increase the E-factor, resulting in a less green process. Whilst maintaining the aldehyde and amine to a 1:1 stoichiometric ratio, excess nitroalkane was necessary since its deprotonation to form the stable nitronate anion is slow and thus an excess of nitroalkane is used to increase the rate of deprotonation. A compromise was found by performing optimisation trials to reduce the amount of excess nitromethane used. Reducing the original 10-fold excess of 3a used up to 5-fold excess resulted in the same conversion rate and a slightly improved yield of $82 \%$ (entry 7). However, further reducing the amount of excess $3 \mathbf{a}$ (entries 8,9 ) resulted in a deleterious effect on both the reaction time and yield. Performing the reaction under an atmosphere of nitrogen resulted in a slower reaction time, with complete conversion occurring only after $60 \mathrm{~h}$ and the isolated yield also decreased (entry 10). This was important to determine that the reaction is insensitive to oxygen, thus ensuring simple conditions. Since the catalytic activity was not highly efficient at room temperature, the reaction was heated at different temperatures to increase the rate of reaction. Heating the reaction at $100^{\circ} \mathrm{C}$ resulted in completion after $2 \mathrm{~h}$ and a very high isolated yield was 
122 obtained (entry 11). Lower temperatures, 80 and $60^{\circ} \mathrm{C}$, resulted in longer reaction times and lower

123 yields (entries 12, 13).

124

125

126

127

128

129

130

131

132

133

134

135

136

137

138

139

140

141

142

143

144

145

146

147

148

149

150

151

\section{Figure 5}

Since a new batch of catalyst was used half way through the optimisation trials, a comparison between the two batches was performed by repeating the reaction at room temperature with the new batch (entry 14). This resulted in similar reaction times and yields indicating that increased copper (I) iodide loading did not have any negative consequences on the activity at room temperature.

The effect of solvent on the multicomponent nitro-Mannich reaction was next examined. Solvents from the three major classes: non-polar, polar aprotic and polar protic solvents were used. Nonpolar solvents (Fig. 6, entries 2, 3) and polar non-protic solvents (entries 4, 5) resulted in a poor conversion whilst polar protic solvents (entries 6,7) exhibited good catalytic efficiencies, with high product yields. Despite this, there was no improvement in yield compared to the solvent less reaction and thus the latter more environmentally-friendly condition was maintained.

Figure 6

\section{Examination of substrate scope: nitro-Mannich Reaction with different aldehydes, amines} and nitroalkanes

The generality of the one-pot nitro-Mannich reaction was explored using a $5 \mathrm{~mol} \% \mathrm{CuI}$ on Amberlyst A-21 catalyst (with a loading of $1.52 \mathrm{mmol} \mathrm{CuI} \mathrm{g}{ }^{-1}$ ), a 5-fold excess of nitroalkane, heating at $100^{\circ} \mathrm{C}$ in the presence of air and using solvent-free conditions. A series of aldehydes, amines and nitroalkanes were subjected to the optimised conditions.

Pleasingly, the reaction was shown to tolerate a wide variety of aromatic aldehydes with substituents on the main ring in moderate to excellent yields (Fig. 7, entries 1-10), whilst aliphatic aldehydes were less reactive and resulted in poor to good yields (entries 11-13). Overall, the reaction times for the successful trials performed at $100^{\circ} \mathrm{C}$ were short, irrespective of whether aromatic or aliphatic aldehydes were used. The electronic property of the substituent has a certain effect on the yield. In fact, aromatic aldehyde $\mathbf{1 f}$ (entry 5), with a strong electron-donating group, resulted in a moderate yield since this results in weak aldehyde and imine electrophiles. It is interesting to note that a moderate electron-donating methoxy group in the para position (entries 2 , 10) resulted in very good to excellent isolated product yields. Despite lowering the 
152 electrophilicity of the aldehydes and imines, the methoxy group facilitates complexation of the

153 Lewis acid $\mathrm{Cu}^{+}$catalyst ions, activating the electrophilic aldehyde for nucleophilic attack by the 154 aniline and the intermediate electrophilic imine for attack by nitromethane [51]. The fact that 155 strong electron-donating group (entry 14) did not form the nitro-Mannich product indicates that 156 there is a balance between the opposite forces exerted by the electrophilicity of the carbon and the 157 coordination of the $\mathrm{Cu}^{+}$ions to the oxygen and nitrogen atoms in the $\mathrm{C}=\mathrm{O}$ and $\mathrm{C}=\mathrm{N}$ bonds, 158 respectively. Electron-withdrawing halogen-substituted benzaldehydes (entries 3, 8, 9) resulted in 159 moderate to good yields, overall lower than the model reaction. Despite making the aldehyde and 160 imine more electrophilic, they slow the activation mechanism of the catalyst. Aliphatic aldehydes 161 resulted into poor yields due to being stronger electron-donating groups and also tend to make the 162 intermediate aliphatic imine more unstable by tautomerisation to the enamine, and hence, undergo 163 side reactions. The scope of the reaction was also tested with two ketones (entries 15, 16); however, 164 no product was collected due to lower electrophilicities and additional steric hindrance.

Figure 7

166

167

168

169

170

171

172

173

174

175

176

177

178

179

180 181

Nitro-Mannich products with variation in the aromatic amine substituent (Fig. 8) resulted overall in short reaction times, with moderate to excellent yields. Both electron-donating $\mathbf{2 b - f}$ and

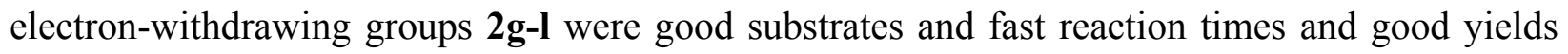
were achieved. Again, such results can be explained in terms of the balance between the increased electron density on the $\mathrm{C}=\mathrm{N}$ bond of the intermediate caused by electron-donating groups, which increased the likelihood of activation by complexation with the Lewis acid $\mathrm{Cu}^{+}$catalyst despite reducing the electrophilicity, and the increase in the electrophilic strength of the imines with electron-withdrawing groups. Aromatic amines $\mathbf{2 f}$ and $\mathbf{2 l}$ resulted in lower yields due to increased steric hindrance. Aliphatic amines (entries 13,14) resulted in no product formation due to the less stable imines and $\beta$-nitroamine products.

\section{Figure 8}

A set of nitro-Mannich reactions (Fig. 9) focused on investigating other nitroalkanes. Increasing the length of the alkyl moiety of the nitroalkane using $\mathbf{3 b}$ and $\mathbf{3 c}$ resulted in an increase in reaction time and a decrease in the isolated yield due to a decrease in the stability of the nitroalkane carbanion. Both reactions afforded a good diastereoselectivity (4:1).

\section{Figure 9}


182 To further shed light on the versatility of the multicomponent nitro-Mannich reaction, substitution

183 of more than one substrate at a time was performed. Overall, the reaction time for the successful 184 trials performed at $100^{\circ} \mathrm{C}$ was fast, between $1.5-4 \mathrm{~h}$, and moderate to excellent yields were 185 obtained in most of trials (Fig. 10). Products $\mathbf{4 b}, \mathbf{4 e}$ and $\mathbf{4 i}$ (entries 2, 5, 9) resulted in short reaction 186 times and high yield, longer reaction times were observed for products $\mathbf{4 g}, \mathbf{4 j}$ and $\mathbf{4 k}$ (entries 7 , $18710,11)$ due to steric hindrance by the bulkier $p$-ethyl moiety and a longer nitroalkane chain. 188 Aliphatic aldehydes $\mathbf{1 m}$ and $\mathbf{1 1}$ resulted in a diminished yield (entries 4, 8). No product was formed 189 due to the bulky ortho and meta positioned nitro moieties in 4 f (entry 6) and 2,6-dichloro190 substituted benzaldehyde $\mathbf{1 j}$ (entry 12). Diastereoselectivity was again observed for products $\mathbf{4 j}$ 191 and $\mathbf{4 k}$, although to a lesser extent.

Figure 10

\section{Mechanistic Aspects}

194 A tentative catalytic cycle is proposed (Fig. 11). The reaction consists of two crucial steps, the 195 condensation step involving imine formation and subsequent nucleophilic addition by a nitroalkane to the in situ formed imine to synthesise the final nitro-Mannich product. In the first step, the Lewis acid metal salt CuI, likely catalyses the nucleophilic addition of amines to aldehydes to form the intermediate hemiaminal. The Lewis acid also likely functions as a dehydrating agent to remove a water molecule, which is eliminated from the hemiaminal [51]. This results in formation of the imine. The second step involves the coordination of $\mathrm{CuI}$ to the imino nitrogen, thus activating the imine by formation of a positively charged nitrogen atom, making it a stronger electrophile. This type of activation is typical of copper catalysts used in the nitroMannich reaction [52]. Moreover, validity of the possible mechanism was shown by the fast reaction times and high yields obtained with electron-donating groups since these resulted in a

205

206

207 208 greater electron density on the $\mathrm{C}=\mathrm{N}$, which helped the activation of the imine by coordinating with the Lewis acid $\mathrm{Cu}^{+}$catalyst. It was suggested that the $\mathrm{CuI}$ salt coordinates also to the nitroalkane oxygen, increasing its acidity and thus favouring deprotonation to form the reactive nitronate anion $[52]$.

\section{Figure 11}

The base deprotonating the nitroalkane was likely to be the Amberlyst A-21 support, since it contains a tertiary amine, which is a much stronger base than iodide anions in CuI. Hence, unchelated dimethylamino sites likely deprotonate the nitroalkane to form the reactive nucleophilic 
213 nitronate species, which attacks the activated imine to form the $\beta$-nitroamine product, and at the

214 same time releasing the catalyst to be used for a next cycle.

215 Environmental Acceptability of the nitro-Mannich Reaction

216 The greenness of the nitro-Mannich reaction was determined using two main parameters: the E-

217 Factor and the atom economy. Being a multicomponent reaction, involving only catalytic amounts

218 of catalyst, and consisting of a condensation and an addition step, which only generates water as a

219 by-product, the developed protocol displays both a very high Atom Economy as well as a low E-

220 Factor (based on the model reaction for the synthesis of 6, Eq. 1 and Eq. 2).

221

$$
E-\text { Factor }_{\text {CuI. } A-21}=\frac{\text { Total Waste }(g)}{\text { Total Product }(g)}=\frac{0.610+0.045}{0.520}=1.26
$$

Equation 1: Low E-factor for the multicomponent nitro-Mannich reaction.

$$
\text { Atom Economy }=\frac{R M M_{\text {product }}}{\sum R M M_{\text {reagents }}} \times 100=\frac{242}{(106+93+61)} \times 100=93 \%
$$

Equation 2: High Atom Economy since only water is formed as by-product.

226

The effectiveness of the heterogeneous catalyst was demonstrated by comparison of the amount of waste generated with unsupported copper(I) iodide (Fig. 12). The results clearly indicate the advantage of using the polymer-supported heterogeneous catalyst, which not only resulted in a much higher isolated yield for $\mathbf{6}$ but also in a lower E-Factor, indicating that the reaction performed using a recoverable heterogeneous catalysis was more environmentally-friendly, in terms of the waste produced.

\section{Figure 12}

Amberlyst A-21 supported copper(I) iodide displays a number of advantages (Fig. 13) which makes it an interesting and effective heterogeneous catalyst in synthetic organic chemistry.

\section{Figure 13}

It is a cheap catalyst since both the metal salt and the dimethylaminomethyl-grafted polymer are commercially available. Preparation of the supported catalyst is simple and involves the drying of Amberlyst A-21 followed by chelation of $\mathrm{CuI}$ on the polymeric support [53]. The chelation process 
239 takes approximately one day. The nano-sized copper salt so obtained, being immobilised on the 240 terminal nitrogen in the dimethylamino moiety within the ion-exchange polymeric resin, results in 241 an improvement in activity since the surface area for catalytic activity is increased and such 242 chelation also prevents oxidation or disproportionation of the copper (I) ion, which would affect 243 negatively its activity [49,54]. Handling of the catalyst is also relatively simple due to the beaded 244 form of the resin and hence, only filtration is required to collect the catalyst after completion of 245 the reaction. Thus, the catalyst was recycled to determine the number of cycles it could be 246 consecutively used. The recycling test was performed on the model reaction for the synthesis of 6.

247 The catalyst was washed with diethyl ether solvent, once recovered, and dried under vacuum for one night. The catalyst was immediately reused on the following day for the next recycling trial.

Figure 14

250

251

252

253

254

255

256

257

258

259

260

261

262

263

264

265

266

267

268

As can be seen (Fig. 14), the catalyst had a consistent performance over eight cycles with only a drop of $10 \%$ in yield. This suggests that the heterogeneous catalyst has a good activity and stability. Quantitative analysis of the stability of the heterogeneous catalyst was performed by atomic absorption spectroscopy (AAS), to determine the amount of copper that leached in the solution throughout each cycle (Fig. 15). The recycling test was repeated using the same batch of catalyst, $1 \mathrm{~mL}$ solution was collected from each cycle and tested to determine the percentage of copper from the catalyst that leached into the solution.

\section{Figure 15}

The results (Fig. 15) show that Amberlyst A-21 supported $\mathrm{CuI}$ is very stable since only $1 \% \mathrm{Cu}$ had leached into the solution after eight cycles. Another advantage of the heterogeneous catalyst is that regeneration with more copper (I) iodide is easy, especially in cases where leaching is high, by mixing the polymeric resin support with more metal salt. This increases the shelf-life of the catalyst. The supported catalyst is also advantageous in that it acts as a bifunctional catalyst since only a few of the terminal nitrogen sites in the dimethylamino moiety of the Amberlyst A-21 support are chelated with the Lewis acid copper(I) iodide during mixing [53]. This means that free base moieties are also present and thus both acidic and basic functionalities are active in this catalyst. This also implicates that the catalyst can catalyse different synthetic protocols.

\section{Conclusions}


269 In summary, an efficient one-pot three-component nitro-Mannich reaction between aldehydes, 270 amines and nitroalkanes using $\mathrm{CuI}$ on Amberlyst A-21 as heterogeneous catalyst was developed.

271 The reaction exhibited a green protocol with a high atom economy of $93 \%$ and a low E-Factor of

2721.26 and afforded a wide scope of $\beta$-nitroamines, with more than 25 novel products, in moderate

273 to excellent yields. A degree of selectivity was also observed towards one of the diastereomers.

274 The catalyst proved to be highly active due to its bifunctional Lewis acidic and Brønsted basic

275 nature, and recycling tests indicated a consistent performance over eight cycles, in which a drop

276 of $10 \%$ in yield was observed. The stability of the catalyst was demonstrated using atomic

277 absorption spectroscopy, showing that only $1 \% \mathrm{Cu}$ had leached in the solution after eight cycles.

278 The wide versatility in the substrate functional groups used offers the possibility to synthesise

279 compounds that are more complex and to develop novel synthetic routes to natural products, 280 pharmaceutical products, or dyes in the chemical, food and textile industries.

281

282 Experimental

283 General

284 All reagents are commercially available (Aldrich, Acros, Alfa Aesar, BDH, Cambrian, Fischer 285 Scientific, Hopkins \& Williams, Labkem, Macherey-Nagel, Merck, Riedel de Haen, Scharlau) and 286 were used without further purification except for molecular sieves $4 \AA$, which were heated to 110 $287{ }^{\circ} \mathrm{C}$, and Amberlyst A-21, which was dried before use. TLC plates made up of silica gel on PET288 foil with a fluorescent indicator, fluorescing at $254 \mathrm{~nm}$ under UV irradiation, were used as a 289 stationary phase for TLC. TLC plates were developed using different mixtures of $n$-hexane and 290 ethyl acetate as the mobile phase. Once developed, the plates were observed under a UV lamp at 291 a wavelength of $254 \mathrm{~nm}$, followed by treatment into an iodine-saturated chamber or oxidised in 292 potassium permanganate. A Shimadzu GC-2010 ® gas chromatography instrument was used to 293 monitor the imine formation, where possible, and to identify the components of the crude mixture. 294 The equipment consisted of flame ionisation detector and a HiCap5 column with 30 m (length) by $2950.32 \mathrm{~mm}$ (internal diameter) by $0.25 \mu \mathrm{m}$ (film thickness) dimensions as the stationary phase. The 296 mobile phase consisted of nitrogen carrier gas. The injection temperature was set at $65-300{ }^{\circ} \mathrm{C}$ at 297 a heating rate of $15{ }^{\circ} \mathrm{C} \mathrm{min}-1 .{ }^{1} \mathrm{H}$ and ${ }^{13} \mathrm{C}$ NMR spectra were obtained using a Bruker Avance III 298 HD® NMR Spectrometer, containing an Ascend 50011.75 Tesla Superconducting Magnet, 299 operating at $500.13 \mathrm{MHz}$ and $125.76 \mathrm{MHz}$ for ${ }^{1} \mathrm{H}$ and ${ }^{13} \mathrm{C}$, respectively. The spectra of the products 
300

301

302

303

304

305

306

307

308

309

310

311

312

313

314

315

316

317

318

319

320

321

322

323

324

325

326

327

328

329

were recorded using a Multinuclear $5 \mathrm{~mm}$ PABBO Probe. ACD/Chemsketch (Freeware) Version 12.01 software was used to interpret the spectra. IR spectroscopy was carried out using a Shimadzu IR Affinity-1 FTIR Spectrometer. Calibration of this instrument was done against a $1602 \mathrm{~cm}-1$ polystyrene absorbance spectrum. Product mass spectra were generated using a Waters ${ }^{\circledR}$ ACQUITY® TQD system with a tandem quadrupole mass spectrometer. Melting points of solid products were determined using a Gallenkamp melting point apparatus fitted with a mercury thermometer. Flame atomic absorption spectroscopy (FAAS) was performed using a contraAA ${ }^{\circledR}$ 700 High Resolution Continuum Source Atomic Absorption Spectrometer.

\section{General Method for the Multicomponent nitro-Mannich Reaction}

The general procedure for the nitro-Mannich reaction was based using the optimal conditions selected in Fig. 5, entry $\mathbf{l}$. To a $25 \mathrm{~mL}$ 2-necked round-bottomed flask, equipped with reflux condenser, $5 \mathrm{~mol} \% \mathrm{CuI}$ on Amberlyst A-21 (with a loading of $1.52 \mathrm{mmol} \mathrm{CuI} \mathrm{g}{ }^{-1}$ ), aldehyde (2.5 $\mathrm{mmol})$ and amine $(2.5 \mathrm{mmol})$ were introduced via a plastic funnel, and stirred at $100^{\circ} \mathrm{C}$ using an oil bath till imine formation was complete. The reaction was followed with using TLC and GC, where possible, at several time intervals. Nitromethane $(12.5 \mathrm{mmol})$ was then added to the same flask and the reaction was monitored by TLC. Upon completion, the reaction was stopped and allowed to cool down to room temperature, where heating was performed, and the catalyst was separated by filtration, using $10 \mathrm{~mL}$ ethyl acetate. The crude reaction was then purified by column chromatography (hexane/ethyl acetate) or by direct recrystallization of the reaction mixture from ethanol, and dried under vacuum. Isolated yields were obtained and the products were analysed by IR, ${ }^{1} \mathrm{H}$ and ${ }^{13} \mathrm{C}$ NMR spectroscopy, and mass spectrometry.

\section{Preparation of Copper(I) Iodide on Amberlyst A-21}

A sample of $20 \mathrm{~g}$ of dry Amberlyst A-21 was first prepared by suspending it in $100 \mathrm{~mL}$ methanol for 30 minutes. After this, the mixture was filtered and washed in methanol for a further three times. This method was repeated in dichloromethane. The resin was then dried on a rotary evaporator at $50^{\circ} \mathrm{C}$ until it was free-flowing and subsequently left overnight in a desiccator under vacuum. The dry weight of Amberlyst A-21 was $9.63 \mathrm{~g}$. Amberlyst-21 (5 g) were then added to a solution of $2.86 \mathrm{~g}$ (15 mmol) copper(I) iodide in $75 \mathrm{~mL}$ acetonitrile and left to stir for $24 \mathrm{~h}$ at room temperature. The solvent was then evaporated and the green resin was washed with two $75 \mathrm{~mL}$ aliquots of acetonitrile, followed by two $75 \mathrm{~mL}$ aliquots of dichloromethane. The resin was then 
330 331 332 333 334 335 336 337 338 339 340 341 342 343

dried on a rotary evaporator at $40^{\circ} \mathrm{C}$ and subsequently left overnight in a desiccator under vacuum. The loading of copper per gram of resin was calculated by observing the weight increases of the dried sample of $\mathrm{CuI}$ on Amberlyst A-21. A weight increase of $2.05 \mathrm{~g}$ (10.8 mmol CuI $)$ was observed, which gave a polymer loading of $1.52 \mathrm{mmol} \mathrm{CuI} \mathrm{g}{ }^{-1}$.

\section{Analytical Data for selected products.}

$6 \mathrm{~N}$-(2-nitro-1-phenylethyl)aniline [55]. Brown solid. m.p. 73-75 ${ }^{\circ} \mathrm{C}$. IR (neat $\mathrm{KBr}$, $\left.\mathrm{cm}^{-1}\right): v=3354,3078,3058,3027,2970,2916,2894,1604,1546,1514,1508,1489,1458,1425$, $1381,1314,1264,1213,1181,1127,1083,1067,1032,994,940,879,851,822,756,727,701$, 654, 628, 533, 511. ${ }^{1} \mathrm{H}$ NMR $\left(\mathrm{CDCl}_{3}, 500 \mathrm{MHz}\right) \delta(\mathrm{ppm}): 4.35$ (d, 1H, Ha, J=6.4 Hz), 4.70 (d, 2H, $\mathrm{Hb}, J=6.8 \mathrm{~Hz}), 5.15(\mathrm{q}, 1 \mathrm{H}, \mathrm{Hc}, J=6.8 \mathrm{~Hz}), 6.60$ (d, 2H, Hd, $\left.J_{\mathrm{o}}=8.6 \mathrm{~Hz}, J_{\mathrm{m}}=1 \mathrm{~Hz}\right), 6.75(\mathrm{t}, 1 \mathrm{H}, \mathrm{He}$, $J=7.4 \mathrm{~Hz}), 7.15$ (t, 2H, Hf, $J=8.0 \mathrm{~Hz}), 7.30$ (m,1H Hg), 7.40 (m, 4H Hh, Hi). ${ }^{13} \mathrm{C} \mathrm{NMR}\left(\mathrm{CDCl}_{3}\right.$, $126 \mathrm{MHz}) \delta(\mathrm{ppm}): 56.7,80.0,113.9,119.0,126.5,128.7,129.3,129.4,137.8,145.7 . \mathrm{MS}(\mathrm{ES}+)$ $\mathrm{m} / \mathrm{z}=242.07\left[\mathrm{MH}^{+}\right], 122.03,94.06,92.04,86.98,92.04$.

7a $N$-(2-nitro-1-( $p$-tolyl)ethyl)aniline [56]. Dark-red thick oil. IR (neat $\left.\mathrm{NaCl}, \mathrm{cm}^{-1}\right): v=3400$, 3091, 3053, 3027, 2954, 2921, 2863, 1633, 1603, 1554, 1506, 1458, 1436, 1423, 1378,1338, 1316, 1266, 1214, 1182, 1157, 1113, 1068, 1031, 1021, 994, 971, 914, 872, 814, 752, 693, 669, 510. ${ }^{1} \mathrm{H}$ NMR $\left(\mathrm{CDCl}_{3}, 500 \mathrm{MHz}\right) \delta(\mathrm{ppm}): 2.33(\mathrm{~s}, 3 \mathrm{H}, \mathrm{Ha}), 4.35$ (d, 1H, Hb, J=6.1 Hz), 4.70 (m, 2H, Hc), 5.15 (q, 1H, Hd, $J=6.7 \mathrm{~Hz}), 6.61$ (d, 2H, He, $J=8.6 \mathrm{~Hz}), 6.75$ (t, 1H, Hf, $J=7.4 \mathrm{~Hz}$ ), 7.14 (t, 2H, $\left.\mathrm{Hg}, J_{\mathrm{o}}=8.0 \mathrm{~Hz}, J_{\mathrm{m}}=1.2 \mathrm{~Hz}\right), 7.18(\mathrm{~d}, 2 \mathrm{H}, \mathrm{Hh}, J=7.9 \mathrm{~Hz}), 7.25(\mathrm{~d}, 2 \mathrm{H}, \mathrm{Hi}, J=8.2 \mathrm{~Hz}) .{ }^{13} \mathrm{C} \mathrm{NMR}$ $\left(\mathrm{CDCl}_{3}, 126 \mathrm{MHz}\right) \delta(\mathrm{ppm}): 21.1,56.5,80.0,113.9,118.9,126.4,129.4,130.0,134.7,138.5$, 145.8. MS (ES+) $\mathrm{m} / \mathrm{z}=257.10\left[\mathrm{MH}^{+}\right], 196.12,164.05,118.06,94.06,91.07$.

7c $N$-(1-(4-bromophenyl)-2-nitroethyl)aniline. Dark-red thick oil. IR (neat $\left.\mathrm{NaCl}, \mathrm{cm}^{-1}\right): v=3404$, $3087,3053,3026,2963,2918,1603,1553,1508,1423,1404,1377,1335,1314,1263,1215,1180$, $1155,1128,1099,1072,1011,993,902,918,876,822,752,692,648,540,509 .{ }^{1} \mathrm{H} \mathrm{NMR}\left(\mathrm{CDCl}_{3}\right.$, $500 \mathrm{MHz}) \delta(\mathrm{ppm}): 4.40(\mathrm{~d}, 1 \mathrm{H}, \mathrm{Ha}, J=6.3 \mathrm{~Hz}), 4.69$ (d, 2H, Hb, $J=6.6 \mathrm{~Hz}), 5.12$ (q, 1H, Hc, $J=6.6$ $\mathrm{Hz}), 6.58$ (d, 2H, Hd, J=7.7 Hz), 6.75 (t, 1H, He, $J=7.4 \mathrm{~Hz}), 7.15$ (t, 2H, Hf $J=7.4 \mathrm{~Hz}$ ), 7.28 (d, $2 \mathrm{H}, \mathrm{Hg}, J=8.5 \mathrm{~Hz}), 7.51(\mathrm{~d}, 2 \mathrm{H}, \mathrm{Hh}, J=8.5 \mathrm{~Hz}) .{ }^{13} \mathrm{C} \mathrm{NMR}\left(\mathrm{CDCl}_{3}, 126 \mathrm{MHz}\right) \delta(\mathrm{ppm}): 56.1,79.7$, $113.9,119.2,122.6,128.2,129.4,132.5,137.8,145.3 . \mathrm{MS}(\mathrm{ES}+) \mathrm{m} / \mathrm{z}=323.04\left[\mathrm{MH}^{+}\right], 262.04$, $183.99,103.02,93.98,77.01$.

7d $\mathrm{N}$-(2-nitro-1-(2-nitrophenyl)ethyl)aniline [57]. Yellow-brown solid. m.p. $77-79^{\circ} \mathrm{C}$. IR (neat, $\left.\mathrm{KBr}, \mathrm{cm}^{-1}\right): v=3375,3104,3078,3030,2920,2862,1622,1580,1560,1522,1474,1435,1386$, 1340, 1289, 1263, 1250, 1215, 1182, 1154, 1131, 1086, 1066, 937, 921, 879, 860, 851, 811, 792, 750, 724, 708, 673, 647, 627, 560, 517, 505. ${ }^{1} \mathrm{H} \mathrm{NMR}\left(\mathrm{CDCl}_{3}, 500 \mathrm{MHz}\right) \delta(\mathrm{ppm}): 4.83(\mathrm{~m}, 2 \mathrm{H}$, 
364

365

366

367

368

369

370

371

372

373

374

375

376

377

378

379

380

381

382

383

384

385

386

387

388

389

390

391

392

393

394

395

396

397

398

399

Ha), $5.01(\mathrm{dd}, 1 \mathrm{H}, \mathrm{Hb} J=3.7 \mathrm{~Hz}, 12.4 \mathrm{~Hz}), 5.80(\mathrm{~m}, 1 \mathrm{H}, \mathrm{Hc}), 6.48\left(\mathrm{~d}, 2 \mathrm{H}, \mathrm{Hd}, J_{\mathrm{o}}=8.6 \mathrm{~Hz}, J_{\mathrm{m}}=1.0\right.$ $\mathrm{Hz}), 6.75\left(\mathrm{t}, 1 \mathrm{H}, \mathrm{He}, J_{\mathrm{o}}=7.4 \mathrm{~Hz}, J_{\mathrm{m}}=1.0 \mathrm{~Hz}\right), 7.10$ (t, 2H, Hf, $\left.J=8.0 \mathrm{~Hz}\right), 7.50$ (t, 1H, Hg, $J_{\mathrm{o}}=8.1$ $\left.\mathrm{Hz}, J_{\mathrm{m}}=1.4 \mathrm{~Hz}\right), 7.60\left(\mathrm{t}, 1 \mathrm{H}, \mathrm{Hh}, J_{\mathrm{o}}=7.5 \mathrm{~Hz}, J_{\mathrm{m}}=1.2 \mathrm{~Hz}\right), 7.69\left(\mathrm{~d}, 1 \mathrm{H}, \mathrm{Hi}, J_{\mathrm{o}}=7.9 \mathrm{~Hz}, J_{\mathrm{m}}=1.3 \mathrm{~Hz}\right)$, $8.09\left(\mathrm{~d}, 1 \mathrm{H}, \mathrm{Hj}, J_{\mathrm{o}}=8.2 \mathrm{~Hz}, J_{\mathrm{m}}=1.2 \mathrm{~Hz}\right)$.

7e 3-(2-Nitro-1-(phenylamino)ethyl)phenol. Dark-red oil. IR (neat $\mathrm{NaCl}, \mathrm{cm}^{-1}$ ): $v=3407,3097$, 3072 , 3036, 2923, 2856, 1613, 1580, 1560, 1509, 1451, 1422, 1380, 1344, 1325, 1289, 1273, 1257 , $1215,1186,1154,1138,1083,1066,1028,996,970,934,918,876,860,844,792,750,718,692$, 669, 643, 611, 518. ${ }^{1} \mathrm{H} \mathrm{NMR}\left(\mathrm{CDCl}_{3}, 500 \mathrm{MHz}\right) \delta(\mathrm{ppm}): 4.38$ (m, 1H, Ha), 4.69 (d, 2H, Hb, J=6.7 $\mathrm{Hz}), 5.01$ (broad, 1H, Hc), 5.11 (q, 1H, Hd, $J=6.1 \mathrm{~Hz}$ ), 6.60 (d, 2H, He, J=8.6 Hz), 6.75 (t, 1H, Hf, $J=7.4 \mathrm{~Hz}), 6.76(\mathrm{~m}, 1 \mathrm{H}, \mathrm{Hg}), 6.85$ (s, 1H, Hh), 6.95 (d, 1H, Hi, J=7.6 Hz), 7.15 (t, 2H, Hj, $J_{\mathrm{o}}=7.0$ $\left.\mathrm{Hz}, J_{\mathrm{m}}=1.2 \mathrm{~Hz}\right), 7.25(\mathrm{t}, 1 \mathrm{H}, \mathrm{Hk}, J=7.9 \mathrm{~Hz}) .{ }^{13} \mathrm{C} \mathrm{NMR}\left(\mathrm{CDCl}_{3}, 126 \mathrm{MHz}\right) \delta(\mathrm{ppm}): 56.4,79.9$, $113.4,113.9,115.7,118.6,119.0,129.4,130.6,139.6,145.6,156.4$. MS (ES+) m/z $=259.10$ $\left[\mathrm{MH}^{+}\right], 212.17,198.06,166.06,120.07,94.05$.

7g $\mathrm{N}$-(1-([1,1-biphenyl]-4-yl)-2-nitroethyl)aniline. Yellow-red solid. m.p. 97-99 ${ }^{\circ} \mathrm{C}$. IR (neat, $\mathrm{KBr}$, $\left.\mathrm{cm}^{-1}\right): v=3398,3055,3030,2960,2918,1603,1549,1511,1486,1476,1460,1441,1422,1381$, $1314,1266,1257,1213,1180,1155,1133,1076,1008,993,920,876,837,768,755,732,695$, 650, 621, 561, 507. ${ }^{1} \mathrm{H}$ NMR $\left(\mathrm{CDCl}_{3}, 500 \mathrm{MHz}\right) \delta(\mathrm{ppm}): 4.42$ (d, 1H, Ha, J=6.4 Hz), 4.75 (d, 2H, $\mathrm{Hb}, J=6.8 \mathrm{~Hz}), 5.22(\mathrm{q}, 1 \mathrm{H}, \mathrm{Hc}, J=6.6 \mathrm{~Hz}), 6.65\left(\mathrm{~d}, 2 \mathrm{H}, \mathrm{Hd}, J_{\mathrm{o}}=8.6 \mathrm{~Hz}, J_{\mathrm{m}}=1.0 \mathrm{~Hz}\right), 6.75(\mathrm{t}, 1 \mathrm{H}$, $\left.\mathrm{He}, J_{\mathrm{o}}=7.4 \mathrm{~Hz}, J_{\mathrm{m}}=1.0 \mathrm{~Hz}\right), 7.16\left(\mathrm{t}, 2 \mathrm{H}, \mathrm{Hf}, J_{\mathrm{o}}=8.0 \mathrm{~Hz}, J_{\mathrm{m}}=1.2 \mathrm{~Hz}\right), 7.35\left(\mathrm{t}, 1 \mathrm{H}, \mathrm{Hg}, J_{\mathrm{o}}=7.4 \mathrm{~Hz}\right.$, $\left.J_{\mathrm{m}}=1.3 \mathrm{~Hz}\right), 7.43(\mathrm{t}, 2 \mathrm{H}, \mathrm{Hh}, J=7.6 \mathrm{~Hz}), 7.47$ (d, 2H, Hi, $\left.J_{\mathrm{o}}=8.1 \mathrm{~Hz}, J_{\mathrm{m}}=1.6 \mathrm{~Hz}\right), 7.55(\mathrm{~d}, 2 \mathrm{H}, \mathrm{Hj}$, $\left.J_{\mathrm{o}}=8.2 \mathrm{~Hz}, J_{\mathrm{m}}=2.0 \mathrm{~Hz}\right), 7.60\left(\mathrm{~d}, 2 \mathrm{H}, \mathrm{Hk}, J_{\mathrm{o}}=8.4 \mathrm{~Hz}, J_{\mathrm{m}}=2.0 \mathrm{~Hz}\right) .{ }^{13} \mathrm{C} \mathrm{NMR}\left(\mathrm{CDCl}_{3}, 126 \mathrm{MHz}\right) \delta$ (ppm): 56.5, 79.9, 114.0, 119.0, 126.9, 127.1, 127.6, 128.0, 128.9, 129.4, 136.7, 140.3, 141.7, 145.7. $\mathrm{MS}(\mathrm{ES}+) \mathrm{m} / \mathrm{z}=327.03\left[\mathrm{MH}^{+}\right], 80.91$.

7h $\mathrm{N}$-(1-(3,4-dichlorophenyl)-2-nitroethyl)aniline. Dark-red thick oil. IR (neat $\left.\mathrm{NaCl}, \mathrm{cm}^{-1}\right): v=$ 3403, 3093, 3054, 3025, 2960, 2922, 2857, 1604, 1556, 1508, 1472, 1423, 1378, 1313, 1262, 1214 , 1194, 1181, 1136, 1075, 1033, 997, 929, 884, 826, 752, 697, 668, 619, 584. ${ }^{1} \mathrm{H} \mathrm{NMR}\left(\mathrm{CDCl}_{3}, 500\right.$ MHz) $\delta(\mathrm{ppm}): 4.43$ (broad, 1H, Ha), 4.68 (d, 2H, Hb, J=6.9 Hz), 5.11 (q, 1H, Hc, J=6.0 Hz), 6.57 $\left(\mathrm{d}, 2 \mathrm{H}, \mathrm{Hd}, J_{\mathrm{o}}=9.1 \mathrm{~Hz}, J_{\mathrm{m}}=0.95 \mathrm{~Hz}\right), 6.78\left(\mathrm{t}, 1 \mathrm{H}, \mathrm{He}, J_{\mathrm{o}}=7.4 \mathrm{~Hz}, J_{\mathrm{m}}=1.0 \mathrm{~Hz}\right), 7.15(\mathrm{t}, 2 \mathrm{H}, \mathrm{Hf}, J=8.0$ $\mathrm{Hz}), 7.25$ (d, 1H, Hg, J=9.3 Hz), 7.45 (d, 1H, Hh, J=8.2 Hz), 7.51 (s, 1H, Hi). ${ }^{13} \mathrm{C} \mathrm{NMR}\left(\mathrm{CDCl}_{3}\right.$, $126 \mathrm{MHz}) \delta$ (ppm): 55.8, 79.6, 114.0, 119.5, 125.8, 128.6, 129.5, 131.6, 132.9, 133.6, 138.1, 145.1. MS (ES+) m/z = 312.97 [MH'], 252.96, 252.02, 173.91, 101.73, 93.98.

7i $\mathrm{N}$-(1-(2,6-dichlorophenyl)-2-nitroethyl)aniline. Red solid. m.p. 96-99 ${ }^{\circ} \mathrm{C}$. IR (neat, $\left.\mathrm{KBr}, \mathrm{cm}^{-1}\right)$ : $v=3388,3061,3049,3033,2982,2928,2855,1603,1581,1555,1508,1495,1457,1441,1381$, $1339,1311,1289,1266,1250,1216,1200,1181,1152,1123,1089,1056,1028,993,923,901$, $885,860,834,793,774,758,726,695,640,621,558,495 .{ }^{1} \mathrm{H} \mathrm{NMR}\left(\mathrm{CDCl}_{3}, 500 \mathrm{MHz}\right) \delta(\mathrm{ppm})$ : 4.73 (dd, 1H, Ha, J=5.2 Hz, 12.3 Hz), 4.95 (d, 1H, Hb, 11.2 Hz), 5.07 (dd, 1H, Hc, 9.9 Hz, 12.3 
$400 \mathrm{~Hz}$ ), 6.29 (ddd, 1H, Hd, $11.2 \mathrm{~Hz}, 10.0 \mathrm{~Hz}, 5.3 \mathrm{~Hz}$ ), 6.74 (m, 3H, He, Hf), 7.13-7.19 (m, 3H, Hg,

401

402

403

404

405

406

407

408

409

410

411

412

413

414

415

416

417

418

419

420

421

422

423

424

425

426

427

428

429

430

431

432

433

434
$\mathrm{Hh}$ ), 7.27-7.38 (m, 2H, Hi). ${ }^{13} \mathrm{C} \mathrm{NMR}\left(\mathrm{CDCl}_{3}, 126 \mathrm{MHz}\right) \delta(\mathrm{ppm}): 53.3,76.5,114.2,119.4,129.4$, 130.2, 132.2, 145.3. MS (ES+) $\mathrm{m} / \mathrm{z}=310.97\left[\mathrm{MH}^{+}\right], 250.02,136.98,124.95,93.33$.

$7 \mathbf{j} N$-(1-(benzo[d] [1,3]dioxol-5-yl)-2-nitroethyl)aniline. Red thick oil. IR (neat $\mathrm{NaCl}, \mathrm{cm}^{-1}$ ): $v=$ $3393,3051,3028,2960,2896,1607,1559,1508,1491,1449,1378,1339,1313,1246,1184,1155$, $1110,1036,997,971,929,871,816,755,733,693,668,639 .{ }^{1} \mathrm{H} \mathrm{NMR}\left(\mathrm{CDCl}_{3}, 500 \mathrm{MHz}\right) \delta$ (ppm): 4.35 (broad, $1 \mathrm{H}, \mathrm{Ha}$ ), 4.66 (d, 2H, Hb, $J=6.7 \mathrm{~Hz}$ ), 5.07 (q, 1H, Hc, $J=6.0 \mathrm{~Hz}$ ), 5.96 (m, 2H, $\mathrm{Hd}), 6.61\left(\mathrm{~d}, 2 \mathrm{H}, \mathrm{He}, J_{\mathrm{o}}=8.6 \mathrm{~Hz}, J_{\mathrm{m}}=1.0 \mathrm{~Hz}\right), 6.75$ (t, 1H, Hf, $\left.J=7.3 \mathrm{~Hz}\right), 6.79(\mathrm{~d}, 1 \mathrm{H}, \mathrm{Hg}, J=8.6$ $\mathrm{Hz}), 6.87(\mathrm{~m}, 2 \mathrm{H}, \mathrm{Hh}, \mathrm{Hi}), 7.15\left(\mathrm{t}, 2 \mathrm{H}, \mathrm{Hj}, J_{\mathrm{o}}=7.4 \mathrm{~Hz}, J_{\mathrm{m}}=2.0 \mathrm{~Hz}\right) .{ }^{13} \mathrm{C} \mathrm{NMR}\left(\mathrm{CDCl}_{3}, 126 \mathrm{MHz}\right) \delta$ (ppm): 56.5, 80.2, 101.4, 106.7, 108.9, 113.9, 119.0, 120.0, 129.4, 131.6, 145.6, 147.9, 148.5. MS $(\mathrm{ES}+) \mathrm{m} / \mathrm{z}=287.10\left[\mathrm{MH}^{+}\right], 194.02,148.03,94.05,91.64,90.99$.

7k $N$-(1-nitropentan-2-yl)aniline. Red oil. IR (neat NaCl, $\mathrm{cm}^{-1}$ ): $v=3367,3055,3017,2965,2931$, $2875,1684,1604,1555,1500,1463,1445,1383,1362,1317,1258,1220,1185,1140,1075,1047$, 994, 922, 839, 756, 692, 669. ${ }^{1} \mathrm{H}$ NMR $\left(\mathrm{CDCl}_{3}, 500 \mathrm{MHz}\right) \delta(\mathrm{ppm}): 0.95$ (t, $\left.3 \mathrm{H}, \mathrm{Ha}, J=7.3 \mathrm{~Hz}\right)$, 1.40-1.70 (m, 4H, Hb, Hc), 3.65 (d, 1H, Hd, $J=7.2 \mathrm{~Hz}$ ), 4.10 (m, 1H, He), 4.40 (dd, 1H, Hf, $J=6.2$ $\mathrm{Hz}, 11.8 \mathrm{~Hz}), 4.50,(\mathrm{dd}, 1 \mathrm{H}, \mathrm{Hg}, J=5.1 \mathrm{~Hz}, 11.8 \mathrm{~Hz}), 6.65\left(\mathrm{~d}, 2 \mathrm{H}, \mathrm{Hh}, J_{\mathrm{o}}=8.6 \mathrm{~Hz}, J_{\mathrm{m}}=0.9 \mathrm{~Hz}\right), 6.75$ $\left(\mathrm{t}, 1 \mathrm{H}, \mathrm{Hi}, J_{\mathrm{o}}=7.4 \mathrm{~Hz}, J_{\mathrm{m}}=0.9 \mathrm{~Hz}\right), 7.20\left(\mathrm{t}, 2 \mathrm{H}, \mathrm{Hj}, J_{\mathrm{o}}=8.0 \mathrm{~Hz}, J_{\mathrm{m}}=1.2 \mathrm{~Hz}\right) \cdot{ }^{13} \mathrm{C} \mathrm{NMR}\left(\mathrm{CDCl}_{3}, 126\right.$ MHz) $\delta(\mathrm{ppm}): 13.8,19.1,35.1,52.0,78.1,113.5,118.7,129.6,146.0 . \mathrm{MS}(\mathrm{ES}+) \mathrm{m} / \mathrm{z}=209.10$ $\left[\mathrm{MH}^{+}\right], 148.11,118.25,106.02,93.08,40.98$.

$71 \mathrm{~N}$-(1-cyclohexyl-2-nitroethyl)aniline. Red oil. (neat NaCl, $\left.\mathrm{cm}^{-1}\right): v=3399,3053,3022,2930$, $2853,1601,1557,1504,1449,1427,1381,1348,1315,1256,1215,1180,1155,1123,1072,1030$, 993, 964, 914, 893, 874, 841, 752, 692, 667, 619, 509. ${ }^{1} \mathrm{H}$ NMR (CDCl3, $\left.500 \mathrm{MHz}\right) \delta: 1.00-1.30$ (m, 5H, Ha, Hb, Hc), 1.55-1.85 (m, 5H, Hd, He, Hf), 1.91 (d, 1H, Hg, J=12.5 Hz), 3.70 (d, 1H, $\mathrm{Hh}, J=9.8 \mathrm{~Hz}$ ), 3.94-4.00 (m, 1H, Hi), 4.45 (dd, $1 \mathrm{H}, \mathrm{Hj} J=6.3 \mathrm{~Hz}, 12.1 \mathrm{~Hz}$ ), 4.55 (dd, 1H, Hk, $J=5.7 \mathrm{~Hz}, 12.1 \mathrm{~Hz}), 6.65(\mathrm{~d}, 2 \mathrm{H}, \mathrm{Hl}, J=7.7 \mathrm{~Hz}), 6.75(\mathrm{t}, 1 \mathrm{H}, \mathrm{Hm}, J=7.3 \mathrm{~Hz}), 7.2\left(\mathrm{t}, 2 \mathrm{H}, \mathrm{Hn}, J_{\mathrm{o}}=7.9\right.$ $\left.\mathrm{Hz}, J_{\mathrm{m}}=1.0 \mathrm{~Hz}\right) .{ }^{13} \mathrm{C} \mathrm{NMR}\left(\mathrm{CDCl}_{3}, 126 \mathrm{MHz}\right) \delta(\mathrm{ppm}): 26.0,26.1,28.3,29.6,40.6,57.0,76.5$, 113.5, 118.4, 129.5, 146.6. MS (ES+) $\mathrm{m} / \mathrm{z}=249.14\left[\mathrm{MH}^{+}\right], 188.17,109.08,106.02,94.71,67.06$.

7m Ethyl 3-nitro-2-(phenylamino)propanoate. Red oil. IR (neat NaCl, $\mathrm{cm}^{-1}$ ): $v=3388,3054,3028$, 2983, 2936, 2905, 2870, 1742, 1605, 1558, 1507, 1447, 1421, 1380, 1313, 1281, 1256, 1215, 1158 , $1100,1078,1053,1018,958,922,875,856,757,693 .{ }^{1} \mathrm{H} \mathrm{NMR}\left(\mathrm{CDCl}_{3}, 500 \mathrm{MHz}\right) \delta(\mathrm{ppm}): 1.29$ (t, 3H, Ha, J=7.1 Hz), 4.29 (m, 2H, Hb), 4.51 (d, 1H, Hc, J=7.7 Hz), 4.64 (m, 1H, Hd), 4.79 (dd, $1 \mathrm{H}, \mathrm{He}, J=5.0 \mathrm{~Hz}, 13.7 \mathrm{~Hz}$ ), 4.87 (dd, 1H, Hf, $J=4.5 \mathrm{~Hz}, 13.7 \mathrm{~Hz}), 6.68$ (d, 2H, Hg, $J=7.7 \mathrm{~Hz}$ ), 6.84 (t, $1 \mathrm{H}, \mathrm{Hh}, J=7.4 \mathrm{~Hz}), 7.22\left(\mathrm{t}, 2 \mathrm{H}, \mathrm{Hi}, J_{\mathrm{o}}=8.0 \mathrm{~Hz}, J_{\mathrm{m}}=1.9 \mathrm{~Hz}\right) .{ }^{13} \mathrm{C} \mathrm{NMR}\left(\mathrm{CDCl}_{3}, 126 \mathrm{MHz}\right)$ $\delta(\mathrm{ppm}): 14.0,54.9,62.7,75.6,113.8,119.7,129.6,145.2,169.5 . \mathrm{MS}(\mathrm{ES}+) \mathrm{m} / \mathrm{z}=239.10\left[\mathrm{MH}^{+}\right]$, 178.11, 150.07, 104.02, 93.02, 77.02. 
435

436

437

438

439

440

441

442

443

444

445

446

447

448

449

450

451

452

453

454

455

456

457

458

459

460

461

462

463

464

465

466

467

468

469

470

8c 3-Methyl- $N$-(2-nitro-1-phenylethyl)aniline. Red thick oil. IR (neat $\mathrm{NaCl}, \mathrm{cm}^{-1}$ ): $v=3396,3027$, 2949, 2919, 2859, 1608, 1591, 1558, 1508, 1491, 1453, 1424, 1380, 1339, 1323, 1269, 1215, 1178 , 1126, 1078, 1032, 998, 970, 926, 847, 768, 699, 668, 624, 592, 527. ${ }^{1} \mathrm{H} \mathrm{NMR}\left(\mathrm{CDCl}_{3}, 500 \mathrm{MHz}\right)$ $\delta$ (ppm): 2.24 (s, 3H, Ha), 4.31 (d, 1H, Hb, J=6.4 Hz), 4.71 (d, 2H, Hc, J=6.8 Hz), 5.17 (q, 1H, $\mathrm{Hd}, J=6.7 \mathrm{~Hz}), 6.41$ (d, 1H, He, $\left.J_{\mathrm{o}}=8.0 \mathrm{~Hz}, J_{\mathrm{m}}=2.2 \mathrm{~Hz}\right), 6.46$ (s, 1H, Hf), 6.58 (d, 1H, Hg, J=7.5 $\mathrm{Hz}$ ), 7.03 (t, 1H, Hh, $J=7.8 \mathrm{~Hz}$ ), 7.31-7.34 (m, 1H, Hi), 7.35-7.41 (m, 4H, Hj, Hk). ${ }^{13} \mathrm{C}$ NMR $\left(\mathrm{CDCl}_{3}, 126 \mathrm{MHz}\right) \delta(\mathrm{ppm}): 21.5,56.6,79.9,110.9,114.8,119.9,126.4,128.6,129.2,129.3$, 137.8, 139.2, 145.7. MS (ES+) m/z = 257.08 [MH+], 196.11, 149.99, 107.98, 104.07, 78.05.

8d 2-Methyl- $N$-(2-nitro-1-phenylethyl)aniline. Red thick oil. IR (neat $\mathrm{NaCl}, \mathrm{cm}^{-1}$ ): $v=3417,3058$, $3024,2967,2915,2853,1636,1608,1588,1554,1508,1481,1455,1423,1377,1345,1313,1263$, 12161189, 1160, 1136, 1074, 1053, 1030, 1003, 987, 968, 923, 844, 751, 701, 653, 621, 592, 536. ${ }^{1} \mathrm{H} \mathrm{NMR}\left(\mathrm{CDCl}_{3}, 500 \mathrm{MHz}\right) \delta(\mathrm{ppm}): 2.23$ (s, 3H, Ha), 4.35 (d, 1H, Hb, J=5.5 Hz), 4.75 (d, 2H, $\mathrm{Hc}, J=6.7 \mathrm{~Hz}), 5.19$ (q, 1H, Hd, $J=6.4 \mathrm{~Hz}), 6.46$ (d, 1H, He, $J=8.0 \mathrm{~Hz}), 6.69$ (t, 1H, Hf, $J_{\mathrm{o}}=7.3 \mathrm{~Hz}$, $\left.J_{\mathrm{m}}=0.8 \mathrm{~Hz}\right), 7.00\left(\mathrm{t}, 1 \mathrm{H}, \mathrm{Hg}, J_{\mathrm{o}}=7.5 \mathrm{~Hz}, J_{\mathrm{m}}=1.0 \mathrm{~Hz}\right), 7.08(\mathrm{~d}, 1 \mathrm{H}, \mathrm{Hh}, J=7.4 \mathrm{~Hz}), 7.31-7.34(\mathrm{~m}, 1 \mathrm{H}$, $\mathrm{Hi}), 7.36-7.42$ (m, 4H, Hj, Hk). ${ }^{13} \mathrm{C} \mathrm{NMR}\left(\mathrm{CDCl}_{3}, 126 \mathrm{MHz}\right) \delta(\mathrm{ppm}): 17.5,56.8,80.3,111.2$, $118.5,123.0,126.4,127.1,128.7,129.4,130.4,137.9,143.7 . \mathrm{MS}(\mathrm{ES}+) \mathrm{m} / \mathrm{z}=257.08\left[\mathrm{MH}^{+}\right]$, $196.11,149.99,107.98,104.07,77.99$.

8e 4-Ethyl- $N$-(2-nitro-1-phenylethyl)aniline. Red thick oil. IR (neat $\mathrm{NaCl}, \mathrm{cm}^{-1}$ ): $v=3400,3062$, 3027,2962 , 2928, 2889, 2870, 1615, 1557, 1521, 1494, 1452, 1426, 1414, 1377, 1343, 1313, 1265 , $1212,1184,1134,1113,1076,1029,1000,968,921,826,767,700,644,628,540 .{ }^{1} \mathrm{H}$ NMR $\left(\mathrm{CDCl}_{3}, 500 \mathrm{MHz}\right) \delta(\mathrm{ppm}): 1.15(\mathrm{t}, 3 \mathrm{H}, \mathrm{Ha}, J=7.6 \mathrm{~Hz}), 2.50(\mathrm{q}, 2 \mathrm{H}, \mathrm{Hb}, J=7.6 \mathrm{~Hz}), 4.27$ (broad, $1 \mathrm{H}, \mathrm{Hc}), 4.70$ (d, 2H, Hd, $J=6.8 \mathrm{~Hz}$ ), 5.14 (broad, 1H, He), 6.55 (d, 2H, Hf, $J=8.5 \mathrm{~Hz}$ ), 6.98 (d, $2 \mathrm{H}, \mathrm{Hg}, J=8.5 \mathrm{~Hz}), 7.32(\mathrm{~m}, 1 \mathrm{H}, \mathrm{Hh}), 7.36-7.41(\mathrm{~m}, 4 \mathrm{H}, \mathrm{Hi}, \mathrm{Hj}) .{ }^{13} \mathrm{C} \mathrm{NMR}\left(\mathrm{CDCl}_{3}, 126 \mathrm{MHz}\right) \delta$ (ppm): 15.8, 27.9, 57.0, 80.0, 114.1, 126.5, 128.6, 128.7, 129.3, 134.8, 138.0, 143.6. MS (ES+) $\mathrm{m} / \mathrm{z}=271.08\left[\mathrm{MH}^{+}\right], 210.11,121.37,104.01,103.69,78.06$.

8h 1-(4-((2-Nitro-1-phenylethyl)amino)phenyl)ethanone. Dark-red thick oil. IR (neat $\left.\mathrm{NaCl}, \mathrm{cm}^{-1}\right)$ : $v=3319,3156,3087,3068,3027,2963,2919,2864,1651,1594,1562,1533,1496,1457,1425$, 1378, 1362, 1356, 1283, 1216, 1181, 1136, 1117, 1073, 1025, 955, 926, 834, 812, 765, 724, 704, 638, 599, 577. ${ }^{1} \mathrm{H} \mathrm{NMR}\left(\mathrm{CDCl}_{3}, 500 \mathrm{MHz}\right) \delta(\mathrm{ppm}): 2.47$ (s, 3H, Ha), $4.75(\mathrm{~m}, 2 \mathrm{H}, \mathrm{Hb}), 4.99(\mathrm{~d}$, $1 \mathrm{H}, \mathrm{Hc}, J=6.7 \mathrm{~Hz}), 5.26$ (q, 1H, Hd, $J=7.3 \mathrm{~Hz}), 6.60$ (d, 2H, He, $J_{\mathrm{o}}=6.9 \mathrm{~Hz}, J_{\mathrm{m}}=1.9 \mathrm{~Hz}$ ), 7.33-7.42 $(\mathrm{m}, 5 \mathrm{H}, \mathrm{Hf}, \mathrm{Hg}, \mathrm{Hh}), 7.79$ (d, 2H, Hi, $\left.J_{\mathrm{o}}=6.9 \mathrm{~Hz}, J_{\mathrm{m}}=1.9 \mathrm{~Hz}\right) .{ }^{13} \mathrm{C} \mathrm{NMR}\left(\mathrm{CDCl}_{3}, 126 \mathrm{MHz}\right) \delta$ (ppm): 26.1, 56.0, 79.8, 112.7, 126.4, 128.2, 129.0, 129.5, 130.7, 136.8, 149.8, 196.5. MS (ES+) $\mathrm{m} / \mathrm{z}=285.10\left[\mathrm{MH}^{+}\right], 224.14,135.09,104.07,93.08,42.99$.

8i 4-((2-Nitro-1-phenylethyl)amino)benzonitrile. Red thick oil. IR (neat $\left.\mathrm{NaCl}, \mathrm{cm}^{-1}\right): v=3337$, 3059, 3027, 3005, 2965, 2915, 2843, 2215, 1605, 1558, 1519, 1494, 1458, 1417, 1377, 1343, 1311, $1278,1217,1179,1139,1102,1074,1028,1001,968,920,825,761,700,668,627,606,546 .{ }^{1} \mathrm{H}$ 
$471 \mathrm{NMR}\left(\mathrm{CDCl}_{3}, 500 \mathrm{MHz}\right) \delta(\mathrm{ppm}): 4.71(\mathrm{dd}, 1 \mathrm{H}, \mathrm{Ha}, J=8.5 \mathrm{~Hz}, 12.5 \mathrm{~Hz}), 4.76(\mathrm{dd}, 1 \mathrm{H}, \mathrm{Hb}, J=$

472

473

474

475

476

477

478

479

480

481

482

483

484

485

486

487

488

489

490

491

492

493

494

495

496

497

498

499

500

501

502

503

504

505

506 $4.7 \mathrm{~Hz}, 12.5 \mathrm{~Hz}$ ), 5.03 (broad d, 1H, Hc, J=6.5 Hz), 5.20 (m, 1H Hd), 6.60 (d, 2H, He, $J_{\mathrm{o}}=8.9 \mathrm{~Hz}$, $\left.J_{\mathrm{m}}=2.0 \mathrm{~Hz}\right), 7.34-7.38(\mathrm{~m}, 3 \mathrm{H}, \mathrm{Hf}, \mathrm{Hg}), 7.38-7.42(\mathrm{~m}, 4 \mathrm{H}, \mathrm{Hh}, \mathrm{Hi}) .{ }^{13} \mathrm{C} \mathrm{NMR}\left(\mathrm{CDCl}_{3}, 126 \mathrm{MHz}\right)$ $\delta$ (ppm): $55.9,79.7,100.7,113.5,120.0,126.3,129.1,129.6,133.8,136.9,149.2 . \mathrm{MS}(\mathrm{ES}+) \mathrm{m} / \mathrm{z}$ $=268.10\left[\mathrm{MH}^{+}\right], 221.12,143.08,118.10,91.05$.

8j 3-Nitro- $N$-(2-nitro-1-phenylethyl)aniline. Red thick oil. IR (neat $\mathrm{NaCl}, \mathrm{cm}^{-1}$ ): $v=3392,3085$, 3066, 3030, 3004, 2971, 2921, 2862, 1623, 1561, 1526, 1507, 1459, 1424, 1379, 1348, 1276, 1220, 1129, 1099, 1081, 998, 964, 890, 853, 817, 793, 761, 737, 700, 670, 624, 529. ${ }^{1} \mathrm{H} \mathrm{NMR}\left(\mathrm{CDCl}_{3}\right.$, $500 \mathrm{MHz}) \delta(\mathrm{ppm}): 4.70-4.75(\mathrm{dd}, 1 \mathrm{H}, \mathrm{Ha}, J=8.7 \mathrm{~Hz}, 12.4 \mathrm{~Hz}), 4.75-4.80(\mathrm{dd}, 1 \mathrm{H}, \mathrm{Hb}, J=4.5 \mathrm{~Hz}$, $12.4 \mathrm{~Hz}), 4.88$ (d, 1H, Hc, $J=6.7 \mathrm{~Hz}), 5.23$ (d, 1H, Hd, J=4.7, 6.6, 8.5 Hz), 6.89 (d, 1H, He, $J_{0}=8.2$ $\mathrm{Hz}, J_{\mathrm{m}}=2.0 \mathrm{~Hz}$ ), 7.25 (t, 1H, Hf, J=8.2 Hz), 7.34-7.36 (m, 1H, Hg), 7.38-7.42 (m, 5H, Hh, Hi, Hj), $7.55\left(\mathrm{~d}, 1 \mathrm{H}, \mathrm{Hk} J_{\mathrm{o}}=8.2 \mathrm{~Hz}, J_{\mathrm{m}}=1.4 \mathrm{~Hz}\right) .{ }^{13} \mathrm{C} \mathrm{NMR}\left(\mathrm{CDCl}_{3}, 126 \mathrm{MHz}\right) \delta(\mathrm{ppm}): 56.4,79.9,108.0$, $113.5,119.6,126.3,129.1,129.6,130.0,136.4,146.6,149.3 . \mathrm{MS}(\mathrm{ES}+) \mathrm{m} / \mathrm{z}=288.08\left[\mathrm{MH}^{+}\right]$, $150.05,138.99,104.06,91.97,77.99$.

8k 2,5-Dichloro- $N$-(2-nitro-1-phenylethyl)aniline. Red thick oil. IR (neat $\mathrm{NaCl}, \mathrm{cm}^{-1}$ ): $v=3483$, 3396, 3090, 3067, 3031, 2963, 2921, 2853, 1594, 1559, 1508, 1485, 1459, 1417, 1378, 1343, 1294, $1211,1136,1094,1046,952,910,835,794,768,700,613,581,532 .{ }^{1} \mathrm{H} \mathrm{NMR}\left(\mathrm{CDCl}_{3}, 500 \mathrm{MHz}\right)$ $\delta(\mathrm{ppm}): 4.75$ (m, 2H, Ha), 5.18 (q, 1H, Hb, J=6.4 Hz), 5.23 (d, 1H, Hc, J=6.9 Hz), 6.51 (s, 1H, $\left.\mathrm{Hd}, J_{\mathrm{m}}=2.3 \mathrm{~Hz}\right), 6.65\left(\mathrm{~d}, 1 \mathrm{H}, \mathrm{He}, J_{\mathrm{o}}=8.4 \mathrm{~Hz}, J_{\mathrm{m}}=2.3 \mathrm{~Hz}\right), 7.18(\mathrm{~d}, 1 \mathrm{H}, \mathrm{Hf}, J=8.4 \mathrm{~Hz}), 7.35-7.42$ (m, 5H, Hg, Hh, Hi). ${ }^{13} \mathrm{C} \mathrm{NMR}\left(\mathrm{CDCl}_{3}, 126 \mathrm{MHz}\right) \delta$ (ppm): 56.3, 79.9, 112.4, 118.2, 118.7, 126.3,

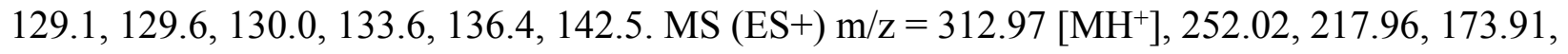
101.73, 93.98 .

$8 \mathbf{l}(E)-N$-(2-nitro-1-phenylethyl)-4-(phenyldiazenyl)aniline. Red solid. m.p. 103-105 ${ }^{\circ} \mathrm{C}$. IR (neat, $\left.\mathrm{KBr}, \mathrm{cm}^{-1}\right): v=3483,3396,3090,3067,3031,2963,2921,2853,1594,1559,1508,1485,1459$, $1417,1378,1343,1294,1211,1136,1094,1046,952,910,835,794,768,700,613,581,532 .{ }^{1} \mathrm{H}$ NMR $\left(\mathrm{CDCl}_{3}, 500 \mathrm{MHz}\right) \delta(\mathrm{ppm}): 4.77(\mathrm{~m}, 1 \mathrm{H}, \mathrm{Ha}), 4.88$ (broad, 1H, Hb), 5.28 (broad, 1H, Hc), $6.69\left(\mathrm{~d}, 2 \mathrm{H}, \mathrm{Hd}, J_{\mathrm{o}}=6.8 \mathrm{~Hz}, J_{\mathrm{m}}=2.1 \mathrm{~Hz}\right), 7.32-7.42(\mathrm{~m}, 6 \mathrm{H}, \mathrm{He}, \mathrm{Hf}, \mathrm{Hg}, \mathrm{Hh}), 7.46$ (t, 2H, Hi, $J_{\mathrm{o}}=7.5$ $\left.\mathrm{Hz}, J_{\mathrm{m}}=1.5 \mathrm{~Hz}\right), 7.79\left(\mathrm{~d}, 2 \mathrm{H}, \mathrm{Hj}, J_{\mathrm{o}}=6.9 \mathrm{~Hz}, J_{\mathrm{m}}=2.1 \mathrm{~Hz}\right), 7.82(\mathrm{~d}, 2 \mathrm{H}, \mathrm{Hk}, J=8.4 \mathrm{~Hz}) .{ }^{13} \mathrm{C} \mathrm{NMR}$ $\left(\mathrm{CDCl}_{3}, 126 \mathrm{MHz}\right) \delta(\mathrm{ppm}): 56.3,79.8,113.6,122.4,125.1,126.4,128.9,129.0,129.5,130.0$, 137.0, 145.8, 148.2, 152.9. MS (ES+) m/z = 347.16 [MH'], 197.07, 104.13, 91.97, 77.07, 65.04.

4a 3-Nitro- $N$-(2-nitro-1-( $p$-tolyl)ethyl)aniline. Red thick oil. IR (neat $\mathrm{NaCl}, \mathrm{cm}^{-1}$ ): $v=3398,3089$, 3027,2923 , 2864, 1624, 1588, 1559, 1536, 1422, 1380, 1351, 1266, 1214, 1181, 1109, 1096, 1071, 1044, 1025, 996, 966, 918, 859, 820, 794, 758, 739, 673, 521. ${ }^{1} \mathrm{H} \mathrm{NMR}\left(\mathrm{CDCl}_{3}, 500 \mathrm{MHz}\right) \delta$ (ppm): 2.34 (s, 3a, Ha), 4.72 (m, 2H, Hb), 4.86 (d, 1H, Hc, J=6.7 Hz), 5.19 (ddd, 1H, Hd, J=8.4 $\mathrm{Hz}, 7.0 \mathrm{~Hz}, 4.7 \mathrm{~Hz}), 6.89$ (d, 1H, He, $\left.J_{\mathrm{o}}=8.2 \mathrm{~Hz}, J_{\mathrm{m}}=0.7 \mathrm{~Hz}\right), 7.2$ (d, 2H, Hf, J=8.9 Hz), 7.24-7.29 $(\mathrm{m}, 3 \mathrm{H}, \mathrm{Hg}, \mathrm{Hh}), 7.41\left(\mathrm{~s}, 1 \mathrm{H}, \mathrm{Hi}, J_{\mathrm{m}}=2.3 \mathrm{~Hz}\right), 7.56\left(\mathrm{~d}, 1 \mathrm{H}, \mathrm{Hj}, J_{\mathrm{o}}=8.1 \mathrm{~Hz}, J_{\mathrm{m}}=0.8 \mathrm{~Hz}\right) .{ }^{13} \mathrm{C} \mathrm{NMR}$ 
$507\left(\mathrm{CDCl}_{3}, 126 \mathrm{MHz}\right) \delta(\mathrm{ppm}): 21.1,56.2,80.0,108.0,113.4,119.6,126.2,130.0,130.2,133.4$,

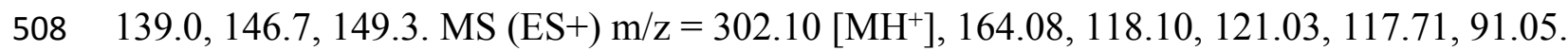

509 4b 4-Chloro- $N$-(1-(4-methoxyphenyl)-2-nitroethyl)aniline. Red thick oil. IR (neat $\left.\mathrm{NaCl}, \mathrm{cm}^{-1}\right): v$ $510=3390,3106,3070,3034,3008,2962,2936,2916,2838,1605,1559,1513,1497,1464,1421$, $5111379,1340,1310,1254,1176,1121,1091,1032,970,826,783,731,682,640,564,548,525$, 512 509. ${ }^{1} \mathrm{H} \mathrm{NMR}\left(\mathrm{CDCl}_{3}, 500 \mathrm{MHz}\right) \delta(\mathrm{ppm}): 3.79$ (s, 3H, Ha), 4.4 (d, 1H, Hb, J=6.4 Hz), 4.67 (d, $5132 \mathrm{H}, \mathrm{Hc}, J=6.7 \mathrm{~Hz}$ ), 5.06 (q, 1H, Hd, $J=6.7 \mathrm{~Hz}$ ), 6.52 (d, 2H, He, $J=8.9 \mathrm{~Hz}), 6.90$ (d, 2H, Hf, $J=8.8$ $514 \mathrm{~Hz}), 7.08$ (d, 2H, Hg, $J=8.9 \mathrm{~Hz}), 7.28$ (d, 2H, Hh, $J=8.6 \mathrm{~Hz}) .{ }^{13} \mathrm{C} \mathrm{NMR}\left(\mathrm{CDCl}_{3}, 126 \mathrm{MHz}\right) \delta(\mathrm{ppm})$ : $51555.3,56.3,80.1,114.8,115.1,127.6,129.2,131.2,139.1,144.3,159.9 . \mathrm{MS}(\mathrm{ES}+) \mathrm{m} / \mathrm{z}=307.09$ $516\left[\mathrm{MH}^{+}\right], 180.05,119.04,91.06$.

517 4c 3-(1-((4-methoxyphenyl)amino)-2-nitroethyl)phenol. Dark-red thick oil. IR (neat $\left.\mathrm{NaCl}, \mathrm{cm}^{-1}\right)$ : $518 v=3361,3306,3066,3035,3000,2952,2931,2827,1591,1560,1513,1457,1377,1298,1239$, $5191183,1127,1035,997,934,827,788,730,699,517 .{ }^{1} \mathrm{H} \mathrm{NMR}\left(\mathrm{CDCl}_{3}, 500 \mathrm{MHz}\right) \delta(\mathrm{ppm}): 3.71$ 520 (s, 3H, Ha), 4.66 (d, 2H, Hb, J=6.7 Hz), 4.74 (m, 1H, Hc), 5.02 (t, 1H, Hd, J=6.7 Hz), 5.16-5.38 521 (broad, 1H, He), 6.57 (d, 2H, Hf, $\left.J_{\mathrm{o}}=8.9 \mathrm{~Hz}, J_{\mathrm{m}}=2.3 \mathrm{~Hz}\right), 6.73\left(\mathrm{~d}, 2 \mathrm{H}, \mathrm{Hg}, J_{\mathrm{o}}=8.9 \mathrm{~Hz}, J_{\mathrm{m}}=2.2 \mathrm{~Hz}\right.$ ), $5226.77\left(\mathrm{~d}, 1 \mathrm{H}, \mathrm{Hh}, J_{\mathrm{o}}=9.9 \mathrm{~Hz}, J_{\mathrm{m}}=0.6 \mathrm{~Hz}\right), 6.85\left(\mathrm{~s}, 1 \mathrm{H}, \mathrm{Hi}, J_{\mathrm{m}}=2.0 \mathrm{~Hz}\right), 6.94(\mathrm{~d}, 1 \mathrm{H}, \mathrm{Hj}, J=8.6 \mathrm{~Hz}$, $\left.523 J_{\mathrm{m}}=1.5 \mathrm{~Hz}\right), 7.24(\mathrm{t}, 1 \mathrm{H}, \mathrm{Hk}, J=7.9 \mathrm{~Hz}) .{ }^{13} \mathrm{C} \mathrm{NMR}\left(\mathrm{CDCl}_{3}, 126 \mathrm{MHz}\right) \delta(\mathrm{ppm}): 55.7,57.5,80.0$, $524114.6,115.0,115.7,117.2,122.4,130.2,130.6,139.7,139.8,144.0,153.0,156.5 . \mathrm{MS}(\mathrm{ES}+) \mathrm{m} / \mathrm{z}$ $525=289.07\left[\mathrm{MH}^{+}\right], 200.15,166.06,123.92$.

526 4d 4-bromo- $N$-(1-cyclohexyl-2-nitroethyl)aniline. Red solid. IR m.p. 74-75 ${ }^{\circ} \mathrm{C}$. IR (neat, $\mathrm{KBr}$, $\mathrm{cm}^{-}$ 527 1): $v=3405,3386,3100,3077,3064,3038,2929,2851,1598,1558,1509,1489,1446,1424$, $1387,1354,1318,1299,1250,1217,1184,1155,1135,1115,1096,1079,1030,1014,1000,971$,

529

530

531

532

533

534

535

536

537

538

539

540

541

542 931, 915, 892, 816, 803, 745, 699, 672, 639, 597, 508, 442, 423. ${ }^{1} \mathrm{H} \mathrm{NMR}\left(\mathrm{CDCl}_{3}, 500 \mathrm{MHz}\right) \delta$ (ppm): 1.00-1.30 (m, 5H, Ha, Hb, Hc), 1.60 (m, 1H, Hd), 1.65-1.85 (m, He, Hf), 1.90 (m, Hg), 3.72 (d, 1H, Hh, J=9.8 Hz), 3.92 (m, 1H, Hi), 4.46, (dd, 1H, Hj, J=6.8 Hz, $12.2 \mathrm{~Hz}), 4.53$ (dd, 1H, $\mathrm{Hk}, J=5.2 \mathrm{~Hz}, 12.2 \mathrm{~Hz}), 6.53$ (d, 2H, Hl, J=7.4 Hz), 7.26 (m, 2H, Hm). ${ }^{13} \mathrm{C} \mathrm{NMR}\left(\mathrm{CDCl}_{3}, 126\right.$ $\mathrm{MHz}) \delta(\mathrm{ppm}): 25.9,26.1,28.9,29.6,40.8,57.2,76.6,110.0,115.0,132.3,145.7 . \mathrm{MS}(\mathrm{ES}+) \mathrm{m} / \mathrm{z}$ $=325.03\left[\mathrm{MH}^{+}\right], 169.93,154.07,136.05,78.89,60.02$.

4e 4-methyl- $N$-(2-nitro-1-(2-nitrophenyl)ethyl)aniline. Yellow-red solid. m.p. $85-87^{\circ} \mathrm{C}$. IR (neat, $\left.\mathrm{KBr}, \mathrm{cm}^{-1}\right): v=3375,3104,3078,3030,2920,2862,1622,1580,1560,1522,1474,1435,1386$, $1340,1289,1263,1250,1215,1182,1154,1131,1086,1066,937,921,879,860,851,811,792$, 750, 724, 708, 673, 647, 627, 560, 517, 505. ${ }^{1} \mathrm{H} \mathrm{NMR}\left(\mathrm{CDCl}_{3}, 500 \mathrm{MHz}\right) \delta(\mathrm{ppm}): 2.18(\mathrm{~s}, 3 \mathrm{H}$, Ha), 4.64-4.86 (broad, 1H, Hb), 4.83 (dd, 1H, Hc, $J=7.6 \mathrm{~Hz}, 12.5 \mathrm{~Hz}$ ), 5.00 (dd, 1H, Hd, J=3.7 Hz, $12.5 \mathrm{~Hz}), 5.76(\mathrm{dd}, 1 \mathrm{H}, \mathrm{He}, J=3.7 \mathrm{~Hz}, 7.6 \mathrm{~Hz}), 6.40$ (d, 2H, Hf, $J=8.5 \mathrm{~Hz}), 6.66(\mathrm{~d}, 2 \mathrm{H}, \mathrm{Hg}$, $J=8.5 \mathrm{~Hz}), 7.49\left(\mathrm{t}, 1 \mathrm{H}, \mathrm{Hh}, J_{\mathrm{o}}=7.8 \mathrm{~Hz}, J_{\mathrm{m}}=1.5 \mathrm{~Hz}\right), 7.60\left(\mathrm{t}, 1 \mathrm{H}, \mathrm{Hi}, J_{\mathrm{o}}=7.6 \mathrm{~Hz}, J_{\mathrm{m}}=1.4 \mathrm{~Hz}\right), 7.68$ $\left(\mathrm{d}, 1 \mathrm{H}, \mathrm{Hj}, J_{\mathrm{o}}=7.8 \mathrm{~Hz}, J_{\mathrm{m}}=1.4 \mathrm{~Hz}\right), 8.08\left(\mathrm{~d}, 1 \mathrm{H}, \mathrm{Hk}, J_{\mathrm{o}}=8.1 \mathrm{~Hz}, J_{\mathrm{m}}=1.4 \mathrm{~Hz}\right) .{ }^{13} \mathrm{C} \mathrm{NMR}\left(\mathrm{CDCl}_{3}, 126\right.$ 
$543 \mathrm{MHz}) \delta(\mathrm{ppm}): 20.4,52.9,79.3,113.8,125.7,128.7,129.0,129.6,130.0,133.5,134.4,142.5$, 544 148.7. $\mathrm{MS}(\mathrm{ES}+) \mathrm{m} / \mathrm{z}=302.10\left[\mathrm{MH}^{+}\right], 241.15,120.05,119.73,107.05$.

545

546

547

548

549

550

551

552

553

554

555

556

557

558

559

560

561

562

563

564

565

566

567

568

569

570

571

572

573

574

575

576

577

578

4g N-(1-(4-bromophenyl)-2-nitroethyl)-4-ethylaniline. Red oil. IR (neat $\left.\mathrm{NaCl}, \mathrm{cm}^{-1}\right): v=3377$, 3086, 3015, 2957, 2919, 2860, 1604, 1582, 1547, 1511, 1478, 1446, 1414, 1369, 1330, 1307, 1259, $1211,1178,1126,1107,1097,1069,1039,1007,962,917,820,759,726,661,648,532 .{ }^{1} \mathrm{H} \mathrm{NMR}$ $\left(\mathrm{CDCl}_{3}, 500 \mathrm{MHz}\right) \delta(\mathrm{ppm}): 1.15$ (t, 3H, Ha, J=7.6 Hz), 2.50 (q, 2H, Hb, J=7.6 Hz), 4.29 (d, 1H, $\mathrm{Hc}, J=6.1 \mathrm{~Hz}$ ), 4.67 (d, 2H, Hd, $J=6.7 \mathrm{~Hz}$ ), 5.09 (q, 1H, He, $J=6.5 \mathrm{~Hz}$ ), 6.52 (d, 2H, Hf, $J_{\mathrm{o}}=8.5 \mathrm{~Hz}$, $\left.J_{\mathrm{m}}=2.0 \mathrm{~Hz}\right), 6.98(\mathrm{~d}, 2 \mathrm{H}, \mathrm{Hg}, J=8.5 \mathrm{~Hz}), 7.28\left(\mathrm{~d}, 2 \mathrm{H}, \mathrm{Hh}, J_{\mathrm{o}}=8.4 \mathrm{~Hz}, J_{\mathrm{m}}=1.7 \mathrm{~Hz}\right), 7.50(\mathrm{~d}, 2 \mathrm{H}, \mathrm{Hi}$, $\left.J_{\mathrm{o}}=8.5 \mathrm{~Hz}, J_{\mathrm{m}}=1.9 \mathrm{~Hz}\right) .{ }^{13} \mathrm{C} \mathrm{NMR}\left(\mathrm{CDCl}_{3}, 126 \mathrm{MHz}\right) \delta(\mathrm{ppm}): 15.8,27.9,56.5,79.8,114.1,122.6$, 128.2, 128.8, 132.5, 135.2, 137.0, 143.2. MS (ES+) m/z $=351.08\left[\mathrm{MH}^{+}\right], 335.77,180.26,90.98$, 64.97.

4h 4-methyl-N-(1-nitropentan-2-yl)aniline. Red oil. IR (neat $\mathrm{NaCl}, \mathrm{cm}^{-1}$ ): $v=3398,3027,2965$, 2933, 2871, 1621, 1588, 1553, 1520, 1468, 1429, 1383, 1354, 1321, 1305, 1259, 1230, 1208, 1188, $1155,1123,1038,993,921,813,735,644,628,511 .{ }^{1} \mathrm{H} \mathrm{NMR}\left(\mathrm{CDCl}_{3}, 500 \mathrm{MHz}\right) \delta(\mathrm{ppm}): 0.95$ (t, 3H, Ha, J=7.3 Hz), 1.38-1.68 (m, 4H, Hb, Hc), 2.24 (s, 3H, Hd), 3.52 (d, 1H, He, J=7.9 Hz), 4.03 (q, 1H, Hf, $J=5.8 \mathrm{~Hz}), 4.41(\mathrm{dd}, 1 \mathrm{H}, \mathrm{Hg}, J=6.1 \mathrm{~Hz}, 11.7 \mathrm{~Hz}), 4.52(\mathrm{dd}, 1 \mathrm{H}, \mathrm{Hh}, J=5.0 \mathrm{~Hz}$, $11.7 \mathrm{~Hz}), 6.58(\mathrm{~d}, 2 \mathrm{H}, \mathrm{Hi}, J=8.4 \mathrm{~Hz}), 7.01(\mathrm{~d}, 2 \mathrm{H}, \mathrm{Hj}, J=8.0 \mathrm{~Hz}) \cdot{ }^{13} \mathrm{C} \mathrm{NMR}\left(\mathrm{CDCl}_{3}, 126 \mathrm{MHz}\right) \delta$ (ppm): 13.8, 19.2, 20.4, 35.1, 52.4, 78.1, 113.8, 128.0, 130.1, 143.7. MS (ES+) $\mathrm{m} / \mathrm{z}=229.07$ $\left[\mathrm{MH}^{+}\right], 199.24,184.74,118.00,91.06$.

4i (E)- $N$-(2-nitro-1-(p-tolyl)ethyl)-4-(phenyldiazenyl)aniline. Dark-red thick oil. IR (neat NaCl, $\left.\mathrm{cm}^{-1}\right): v=3397,3064,3035,2956,2924,2860,1605,1558,1516,1460,1434,1411,1377,1339$, 1313, 1278, 1240, 1186, 1142, 1100, 1075, 1024, 967, 919, 862, 834, 767, 722, 687, 668, 640, 548, 532. ${ }^{1} \mathrm{H}$ NMR $\left(\mathrm{CDCl}_{3}, 500 \mathrm{MHz}\right) \delta(\mathrm{ppm}): 2.34$ (s, 3H, Ha), 4.75 (d, Hb, 2H, J=6.6 Hz), 4.84 (d, Hc, 1H, J=6.7 Hz), 5.24 (m, Hd, 1H), 6.69 (d, 2H, He, J=8.9 Hz), 7.20 (d, 2H, Hf, J=7.9 Hz), $7.28(\mathrm{~d}, 2 \mathrm{H}, \mathrm{Hg}, J=8.1 \mathrm{~Hz}), 7.39\left(\mathrm{t}, 1 \mathrm{H}, \mathrm{Hh}, J_{\mathrm{o}}=7.3 \mathrm{~Hz}, J_{\mathrm{m}}=1.3 \mathrm{~Hz}\right), 7.46$ (t, 2H, Hi, J=7.9 Hz), $7.79\left(\mathrm{~d}, 2 \mathrm{H}, \mathrm{Hj}, J_{\mathrm{o}}=8.9 \mathrm{~Hz}, J_{\mathrm{m}}=2.0 \mathrm{~Hz}\right), 7.81(\mathrm{~d}, 2 \mathrm{H}, \mathrm{Hk}, J=8.3 \mathrm{~Hz}) .{ }^{13} \mathrm{C} \mathrm{NMR}\left(\mathrm{CDCl}_{3}, 126 \mathrm{MHz}\right)$ $\delta$ (ppm): 21.1, 56.1, 79.9, 113.5, 122.4, 125.1, 126.3, 129.0, 129.9, 130.1, 133.9, 138.8, 145.7, 148.3, 153.0. $\mathrm{MS}(\mathrm{ES}+) \mathrm{m} / \mathrm{z}=361.18\left[\mathrm{MH}^{+}\right], 197.18,118.04,91.90,77.00,64.97$.

4j 1-(4-((1-(4-methoxyphenyl)-2-nitropropyl)amino)phenyl)ethanone. Yellow-red thick oil. IR $\left(\right.$ neat $\left.\mathrm{NaCl}, \mathrm{cm}^{-1}\right): v=3348,3059,3004,2962,2939,2906,2839,1656,1602,1556,1514,1458$, $1439,1426,1388,1365,1307,1281,1254,1184,1147,1122,1083,1034,960,876,833,759$, 669, 636, 597, 532. ${ }^{1} \mathrm{H} \mathrm{NMR}\left(\mathrm{CDCl}_{3}, 500 \mathrm{MHz}\right) \delta(\mathrm{ppm}): 1.56(\mathrm{~d}, 3 \mathrm{H}, \mathrm{Ha}, J=6.8 \mathrm{~Hz}), 2.46(\mathrm{~s}, 3 \mathrm{H}$, $\mathrm{Hb}$ ), 3.79 (s, 3H, Hc), 4.80 (t, 1H, Hd, J= $7.9 \mathrm{~Hz}$ ), 4.99 (m, 1H, He), 5.05 (d, 1H, Hf, J=6.8 Hz), $6.56(\mathrm{~d}, 2 \mathrm{H}, \mathrm{Hg}, J=8.9 \mathrm{~Hz}), 6.89$ (d, 2H, Hh, J=8.8 Hz), 7.21 (d, 2H, Hi, J=8.7 Hz), 7.74 (d, 2H, $\mathrm{Hj}, J=8.8 \mathrm{~Hz}) .{ }^{13} \mathrm{C} \mathrm{NMR}\left(\mathrm{CDCl}_{3}, 126 \mathrm{MHz}\right) \delta(\mathrm{ppm}): 14.1,26.1,55.3,59.7,86.3,112.8,114.6$, 128.0, 130.5, 150.1, 159.8, 196.4. ${ }^{1} \mathrm{H}$ NMR $(\mathrm{CDCl} 3,500 \mathrm{MHz}) \delta(\mathrm{ppm}): 1.52(\mathrm{~d}, \mathrm{Ha}, 3 \mathrm{H}, J=6.5$ 
$579 \mathrm{~Hz}), 2.45(\mathrm{~s}, \mathrm{Hb}, 3 \mathrm{H}), 3.78(\mathrm{~s}, \mathrm{Hc}, 3 \mathrm{H}), 4.85$ (t, 1H, Hd, J=7.8 Hz), $4.94(\mathrm{~m}, 1 \mathrm{H}, \mathrm{He}), 5.05$ (d, $1 \mathrm{H}$, $580 \mathrm{Hf}, J=6.8 \mathrm{~Hz}), 6.54(\mathrm{~d}, 2 \mathrm{H}, \mathrm{Hg}, J=8.9 \mathrm{~Hz}), 6.88(\mathrm{~d}, 2 \mathrm{H}, \mathrm{Hh}, J=8.9 \mathrm{~Hz}), 7.23$ (d, 2H, Hi, J=8.6 $581 \mathrm{~Hz}), 7.76(\mathrm{~d}, 2 \mathrm{H}, \mathrm{Hj}, J=8.8 \mathrm{~Hz}) .{ }^{13} \mathrm{C} \mathrm{NMR}\left(\mathrm{CDCl}_{3}, 126 \mathrm{MHz}\right) \delta(\mathrm{ppm}): 17.1,26.0,55.3,60.3,87.5$, $582112.7,114.7,128.0,130.5,150.1,159.9,196.4$. MS $(\mathrm{ES}+) \mathrm{m} / \mathrm{z}=329.10\left[\mathrm{MH}^{+}\right], 254.11,148.03$, $583136.00,117.01,105.04$.

584 4k 4-methyl- $N$-(2-nitro-1-(2-nitrophenyl)butyl)aniline. Red thick oil. IR (neat $\mathrm{NaCl}$, $\left.585 \mathrm{~cm}^{-1}\right): v=3397,3054,3025,2978,2936,2920,2876,1621,1593,1555,1523,1488,1456,1405$, $5861377,1332,1329,1300,1269,1256,1186,1173,1142,1110,1075,1014,935,884,808,760$, $587716,633,541 .{ }^{1} \mathrm{H} \mathrm{NMR}\left(\mathrm{CDCl}_{3}, 500 \mathrm{MHz}\right) \delta(\mathrm{ppm}): 0.94$ (t, 3H, Ha, J=7.4 Hz), 1.66 (m, 1H, Hb), 5882.08 (m, 1H, Hc), 2.2 (s, 3H, Hd), 4.46 (broad, 1H, He), 4.63 (m, 1H, Hf), 4.73 (m, 1H, Hg), 6.45 589 (d, 2H, Hh, J=8.5 Hz), 6.90 (d, 2H, Hi, J=8.7 Hz), 7.18 (m, 2H, Hj), 7.46 (m, 2H, Hk). ${ }^{13} \mathrm{C}$ NMR $590\left(\mathrm{CDCl}_{3}, 126 \mathrm{MHz}\right) \delta(\mathrm{ppm}): 10.4,20.4,24.8,60.2,94.6,114.1,122.4,128.1,128.6,129.8,132.3$, 591 137.4, 143.2. ${ }^{1} \mathrm{H} \mathrm{NMR}^{*}(\mathrm{CDCl} 3,500 \mathrm{MHz}) \delta(\mathrm{ppm}): 0.97$ (t, 3H, Ha, J=7.3 Hz), 1.90 (m, 1H, Hb), 5922.14 (m, 1H, Hc), 4.24 (broad, 1H, Hd), 4.68 (m, 1H, He), 4.80 (m, 1H, Hf), 6.44 (d, 2H, Hg, J=8.5 $593 \mathrm{~Hz}), 6.92$ (d, 2H, Hh, J=9.1 Hz), 7.20 (m, 2H, Hi), $7.46(\mathrm{~m}, 2 \mathrm{H}, \mathrm{Hj}) .{ }^{13} \mathrm{C} \mathrm{NMR}\left(\mathrm{CDCl}_{3}, 126 \mathrm{MHz}\right)$ $594 \delta$ (ppm): 10.7, 22.4, 24.8, 60.4, 93.7, 114.2, 122.4, 128.4, 128.6, 129.8, 132.2, 137.0, 143.4. MS $595(\mathrm{ES}+) \mathrm{m} / \mathrm{z}=314.12,274.11,210.04,178.06,131.10,108.09,93.04,91.03,60.81$.

\section{Supporting Information}

597 Copies of spectra used in the characterization of compounds are reproduced in the Supporting 598 Information File 1. Analytical Data for all products are reported in the Supporting Information 599 File 2.

\section{Acknowledgements}

601 The authors thank the University of Malta for the equipment and Prof. Robert M. Borg for 602 assistance with the acquisition of the NMR spectra.

\section{References}

604

1. Kolb, H. C.; Finn, M. G.; Sharpless, K. B. 2001. Click Chemistry: Diverse Chemical Function

605

606 from a few Good Reactions. Angew. Chem. Int. Ed., 40:2004-2021.

607

2. Noble, A.; Anderson, J. C. 2013. Nitro-Mannich Reaction. Chem. Rev., 113:2887-2939.

3. El-Sayed, M. T.; Mahmoud, K.; Hilgeroth, A. 2013. Synthesis of $\beta$-Nitroamines via Classical Mannich and Aza-Henry Reactions. Curr. Org. Chem., 17:1200-1224.

4. Anderson, J. C.; Koovits, P. J. 2013. An Enantioselective Tandem Reduction/nitro-Mannich Reaction of Nitroalkenes using a Simple Thiourea Organocatalyst. Chem. Sci., 4:2897-2901.

5. Marqués-López, E.; Merino, P.; Tejero, T.; Herrera, R. P. 2009. Catalytic Enantioselective Aza-Henry Reactions. Eur. J. Org. Chem., 15:2401-2420.

6. Westermann, B. 2003. Asymmetric Catalytic Aza-Henry Reactions Leading to 1,2-Diamines and 1,2-Diaminocarboxylic Acids. Angew. Chem. Int. Ed., 42:151-153. 
615

616

617

618

619

620

621

622

623

624

625

626

627

628

629

630

631

632

633

634

635

636

637

638

639

640

641

642

643

644

645

646

647

648

649

650

651

652

653

654

655

656

657

658

7. Ballini, R.; Petrini M. 2004. Recent Synthetic Developments in the Nitro to Carbonyl Conversion (Nef Reaction). Tetrahedron, 60:1017-1047.

8. Shen, B.; Makley, D. M.; Johnston, J. N. 2010. Umpolung Reactivity in Amide and Peptide Synthesis. Nature, 465:1027-1032.

9. Ruano, J. L. G.; López-Cantarero, J.; de Haro, T.; Alemán, J.; Cid, M. B. 2006. Preparation of $\alpha$-Amino Ketones, $\beta$-Amino Hydroxylamines using Asymmetric aza-Henry Reactions of N-p-Tolylsulfinylimines with Nitroethane. Tetrahedron, 62:12197-12203.

10. Czekelius, C.; Carreira, E. M. 2005. Convenient Transformation of Optically Active Nitroalkanes into Chiral Aldoximes and Nitriles. Angew. Chem. Int. Ed., 44:612-615.

11. Tsuritani, N.; Yamada, K.; Yoshikawa, N.; Shibasaki, M. 2002. Catalytic Asymmetric Syntheses of ICI-199441 and CP-99994 using nitro-Mannich Reaction. Chem. Lett., 276-277.

12. Kumaraswamy, G.; Pitchaiah, A. 2011. A Concise Enantioselective Synthesis of L-(-)733,061 and (2S,3S)-Methyl 3-Aminopiperidine-2-Carboxylate using Catalytic Enantioselective aza-Henry Reaction as Key Step. Tetrahedron, 67:2536-2541.

13. Jakubec, P.; Hawkins, A.; Felzmann, W.; Dixon, D. J. 2012. Total Synthesis of Manzamine A and Related Alkaloids. J. Am. Chem. Soc., 134:17482-17485.

14. Handa, S.; Gnanadesikan, V.; Matsunga, S.; Shibasaki, M. 2010. Heterobimetallic Transition Metal/Rare Earth Metal Bifunctional Catalysis: A Cu/Sm/Schiff Base Complex for SynSelective Catalytic Asymmetric Nitro-Mannich Reaction. J. Am. Chem. Soc., 132:4925-4934.

15. Davis, T. A.; Danneman, M. W.; Johnston, J. N. 2012. Chiral Proton Catalysis of Secondary Nitroalkane Additions to Azomethine: Synthesis of a Potent GlyT1 Inhibitor. Chem. Commun., 48:5578-5580.

16. Weng, J.; Li, Y.-B.; Wang, R.-B.; Li, F.-Q.; Liu, C.; Chan, A. S. C.; Lu, G. 2010. A Practical and Azide-Free Synthetic Approach to Oseltamivir from Diethyl D-Tartrate. J. Org. Chem., 75:3125-3128.

17. Davis, T. A.; Johnston, J. N. 2011. Catalytic, Enantioselective Synthesis of Stilbene cisDiamines: a Concise Preparation of (-)-Nutlin-3, a Potent p53/MDM2 Inhibitor. Chem. Sci., 2:1076-1079.

18. Vara, B. A.; Mayasundari, A.; Tellis, J. C.; Danneman, M. W.; Arredondo, V.; Davis, T. A.; Min, J.; Finch, K.; Guy, R. K.; Johnston, J. N. 2014. Organocatalytic, Diastereo-and Enantioselective Synthesis of Nonsymmetric cis-Stilbene Diamines: A Platform for the Preparation of Single-Enantiomer cis-Imidazolines for Protein-Protein Inhibition. J. Org. Chem., 79:6913-6938.

19. Xie, H.; Zhang, Y.; Zhang, S.; Chen, X.; Wang, W. 2011. Bifunctional Cinchona Alkaloid Thiourea Catalyzed Highly Efficient, Enantioselective Aza-Henry Reaction of Cyclic Trifluoromethyl Ketimines: Synthesis of Anti-HIV Drug DPC 083. Angew. Chem. Int. Ed., 123:11977-11980.

20. Choudhary, M. K.; Das, A.; Kureshy, R. I.; Kumar, M.; Noorul, H. K.; Abdi, S. H.; Bajaj, H. C. 2014. Chiral $\mathrm{Cu}$ (II)-Amino Alcohol Based Complexes for Asymmetric aza-Henry Reaction of N-Ts Imines. Catal. Sci. Technol., 4:548-555.

21. Hynes, P. S.; Stupple, P. A.; Dixon, D. J. 2008. Organocatalytic Asymmetric Total Synthesis of (R)-Rolipram and Formal Synthesis of (3S, 4R)-Paroxetine. Org. Lett., 10:1389-1391.

22. Jakubec, P.; Cockfield, D. M.; Dixon, D. J. 2009. Total Synthesis of (-)-Nakadomarin A. J. Am. Chem. Soc., 131:16632-16633. 
659

660

661

662

663

664

665

666

667

668

669

670

671

672

673

674

675

676

677

678

679

680

681

682

683

684

685

686

687

688

689

690

691

692

693

694

695

696

697

698

699

700

701

702

703

23. Kumaraswamy, G.; Pitchaiah, A. 2011. Highly Enantioselective Synthesis of Orthogonally Protected (2S)-2,3-Diaminopropanoates through Catalytic Phase-Transfer Aza-Henry Reaction. Helv. Chim. Acta, 94:1543-1550.

24. Walvoord, R. R.; Kozlowski, M. C. 2015. Cinchonidinium Acetate as a Convenient Catalyst for the Asymmetric Synthesis of cis-Stilbenediamines. Tetrahedron Lett., 56:3070-3074.

25. Bernardi, L.; Bonini, B. F.; Dessole, G.; Fochi, M.; Comes-Franchini, M.; Gavioli, S.; Ricci, A.; Varchi, G. 2003. Concise and Stereocontrolled Synthesis of Pseudo-C2-symmetric Diamino Alcohols and Triamines for Use in HIV Protease Inhibitors. J. Org. Chem., 68:14181425.

26. Anderson, J. C.; Blake, A. J.; Howell, G. P.; Wilson, C. 2005. Scope and Limitations of the nitro-Mannich Reaction for the Stereoselective Synthesis of 1, 2-Diamines J. Org. Chem., 70:549-555.

27. Pelagalli, A.; Pellacani, L.; Scandozza, E.; Fioravanti, S. 2016. Aza-Henry Reactions on CAlkyl Substituted Aldimines. Molecules, 21:723-823.

28. Adams, H.; Anderson, J. C.; Peace, S.; Pennel, A. M. K. 1998. The nitro-Mannich Reaction and its Application to the Stereoselective Synthesis of 1, 2-diamines. J. Org. Chem., 63:99329934.

29. Rodríguez-Solla , H.; Concellon, C.; Alvaredo, N.; Soengas R. G. 2012. The Use of Samarium or Sodium Iodide Salts as an Alternative for the aza-Henry Reaction. Tetrahedron, 68:17361744.

30. Wang, Y.-H.; Liu, Y.-L.; Cao, Z.-Y.; Zhou, J. 2014. Aza-Henry Reaction of Ketimines Catalyzed by Guanidine and Phosphazene Bases. Asian J. Org. Chem., 3:429-432.

31. Kutovaya, I. V.; Shmatova, O. I.; Tkachuk, V. M.; Melnichenko, N. V.; Vovk, M. V.; Nenajdenko, V. G. 2015. Aza-Henry Reaction with $\mathrm{CF}_{3}$-Ketimines: An Efficient Approach to Trifluoromethylated $\beta$-Nitroamines, 1,2-Diamines, $\alpha$-Aminooximes, and Imidazolidinones. Eur. J. Org. Chem., 30:6749-6761.

32. Ballini, R.; Gabrielli, S.; Palmieri, A.; Petrini, M. 2014. Reaction of $\alpha$-Amido Sulfones with Functionalized Nitrocompounds: a New Two-Step Synthesis of N-Alkoxycarbonyl-2,5Disubstituted Pyrroles. RSC Adv., 4:43258-43261.

33. Komura, K.; Taninaka, Y.; Ohtaki, Y.; Sugi, Y. 2010. H-Y Zeolite is a Versatile Heterogeneous Catalyst for the Synthesis of $\beta$-Nitroamines. Appl. Catal., A, 388:211-215.

34. Chakrapani, L.; Kantam, M. L. 2011. Synthesis of $\beta$-Nitroamines Using Nanocrystalline MgO. Synth. Commun., 41:3442-3447.

35. Soengas, R. G.; Silva, A. M. S. 2013. One-Pot Three-Component Barbier-Type Reaction for the Synthesis of $\beta$-Nitroamines. Synlett, 24:1949-1952.

36. Piscopo, C. G.; Sartori, G.; Mayoral, J. A.; Lanari, D.; Vaccaro, L.; Maggi, R. 2013. Study on the Influence of a Sustainable Medium for the Design of Multistep Processes: ThreeComponent Synthesis of 2-Nitroamines. Synlett, 2596-2600.

37. Cruz-Acosta, F.; de Armas, P.; Garcia-Tellado, F. 2013. Water-Compatible Hydrogen-Bond Activation: A Scalable and Organocatalytic Model for the Stereoselective Multicomponent Aza-Henry Reaction. Chem. Eur. J., 19:16550-16554.

38. Sharma, Y. C.; Singh, B. 2011. Advancements in Solid Acid Catalysts for Ecofriendly and Economically Viable Synthesis of Biodiesel. Biofuels, Bioprod. Bioref., 5:69-92.

39. Bosica, G.; Abdilla, R. 2017. A regioselective one-pot aza-Friedel-Crafts reaction for primary, secondary and tertiary anilines using a heterogeneous catalyst. Green Chem. 19:5683-5690. 
704

705

706

707

708

709

710

711

712

713

714

715

716

717

718

719

720

721

722

723

724

725

726

727

728

729

730

731

732

733

734

735

736

737

738

739

740

741

742

743

744

745

746

747

748

40. Wang, L.; Tan, C.; Liu, X.; Feng, X. 2008. Aza-Henry Reaction of Ketoimines Catalyzed by $\mathrm{Na}_{2} \mathrm{CO}_{3}$ : An Efficient Way to $\beta$-Nitroamines. Synlett, 13:2075-2077.

41. Zhang, F.; Liu, Z.-L.; Liu, J.-T. 2011. Asymmetric aza-Henry Reaction of Chiral Fluoroalkyl $\alpha, \beta$-Unsaturated N-tert-Butanesulfinyl Ketoimines: an Efficient Approach to Enantiopure Fluoroalkylated $\alpha, \beta$-Diamines and $\alpha, \beta$-Diamino Acids. Org. Biomol. Chem., 9:3625-3628.

42. García-Muñoz, M. J.; Dema, H. K.; Foubelo, F.; Yus, M. 2014. Base-Promoted Diastereoselective Addition of Nitromethane and Nitroethane to N-tert-Butylsulfinyl Imines: Synthesis of N-Protected $\alpha$-Amino Acids and Amino Ketones. Tetrahedron: Asymmetry, 25:362-372.

43. Mahasneh, A. S. Z. 2006. Synthesis of Some 2-Aminonitroethanes via Silica Gel-Catalyzed nitro-Mannich Reaction. Naturforsch., 61b:1162-1165.

44. Baricordi, N.; Benetti, S.; Biondini, G.; De Risi, C.; Pollini, G. P. 2004. A New 'One-Pot' Synthesis of 2-Substituted 3-Nitro Pyrrolidines through a Multicomponent Domino Reaction. Tetrahedron Lett., 45:1373-1375.

45. Harichandran, G.; Amalraj, S. D.; Shanmugam, P. 2016. Amberlite IRA-400 Cl Resin Catalyzed Synthesis of Secondary Amines and Transformation into N-((1H-indol-3-yl) (heteroaryl) methyl)-N-heteroaryl benzenamines and bis-Indoles via Multicomponent Reaction. J. Saudi Chem. Soc., 1-10.

46. Arunkumar, R.; Subramani, K.; Ravichandran, S. 2010. Montmorillonite K-10 Clay-Catalyzed Microwave Synthesis of some Mannich Bases and their Characterization. Int. J. ChemTech Res., 2:278-281.

47. Vekariya, R. H.; Prajapati, N. P.; Patel, H. D. 2016. Silica-Supported Polyphosphoric Acid (PPA-SiO ${ }_{2}$ ): An Efficient and Reusable Heterogeneous Catalyst for Ecofriendly Organic Synthesis. Synth. Commun., 46:197-219.

48. Ballini, R.; Bosica, G.; Forconi, P. 1996. Nitroaldol (Henry) Reaction Catalyzed by Amberlyst A-21 as a far Superior Heterogeneous Catalyst. Tetrahedron, 52:1677-1684.

49. Bosica, G.; Gabarretta, J. 2015. Unprecedented One-Pot Multicomponent Synthesis of Propargylamines using Amberlyst A-21 Supported CuI Under Solvent-Free Conditions. RSC Adv., 5:46074-46087.

50. Bosica, G.; Abdilla, R. 2017. The $\mathrm{KA}^{2}$ coupling reaction under green, solventless, heterogeneous catalysis. J. Mol. Catal. A: Chemical, 426:542-549.

51. Dalpozzo, R.; De Nino, A.; Nardi, M.; Russo, B.; Procopio, A. 2006. Erbium(III) Triflate: a Valuable Catalyst for the Synthesis of Aldimines, Ketimines, and Enaminones. Synthesis, No. 7:1127-1132.

52. Das, A.; Choudhary, M. K.; Kureshy, R. I.; Roy, T.; Khan, N. U. H.; Abdi, S. H.; Bajaj, H. C. 2014. Enantioselective Henry and Aza-Henry Reaction in the Synthesis of (R)-Tembamide Using Efficient, Recyclable Polymeric CuII Complexes as Catalyst. ChemPlusChem, 79:1138-1146.

53. Girard, C.; Onen, E.; Aufort, M.; Beauviere, S.; Samson, E.; Hercovic, J. 2006. Reusable Polymer-Supported Catalyst for the [3+2] Huisgen Cycloaddition in Automation Protocols. Org. Lett., 8:1689-1692.

54. Keshavarz, M.; Iravani, N.; Ghaedi, A.; Ahmady, A. Z.; Vafaei-Nezhad, M.; Karimi, S. 2013. Macroporous Polymer Supported Azide and Nanocopper (I): Efficient and Reusable Reagent and Catalyst for Multicomponent Click Synthesis of 1,4-Disubstituted-1H-1,2,3-Triazoles from Benzyl Halides. SpringerPlus, 2:64-71. 
749 55. Choudhary, G.; Peddinti, R. K. 2011. An Expeditious, Highly Efficient, Catalyst-Free and Solvent-Free Synthesis of Nitroamines and Nitrosulfides by Michael Addition. Green Chem., 13:276-282.

752

56. Yang, L.; Xia, C.; Huang, H. 2011. Chiral Phosphoric Acid Catalyzed Enantioselective azaMichael Addition of Aromatic Amines to Nitroolefins. Chin. J. Chem., 32:1573-1576.

753

57. Tajbakhsh, M.; Farhang, M.; Hosseini, A. A. 2014. MgO Nanoparticles as an Efficient and

755 Reusable Catalyst for aza-Michael Reaction. J. Iran. Chem. Soc., 11:665-672.

756 


\section{Figure 1 (on next page)}

Mannich reaction between aldehydes, amines and ketones. 


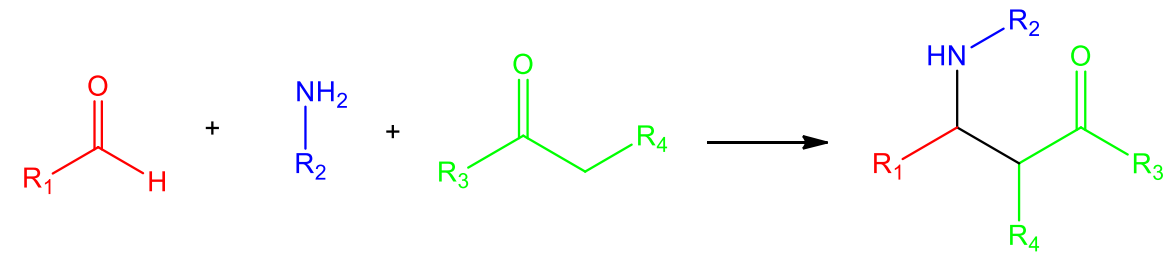

$\mathrm{R}_{1}, \mathrm{R}_{2}, \mathrm{R}_{3}, \mathrm{R}_{4}=\mathrm{H}$, alkyl, aryl 
Figure 2 (on next page)

One-pot multicomponent nitro-Mannich reaction between aldehydes or ketones 1 , amines 2 and nitroalkanes 3 . 


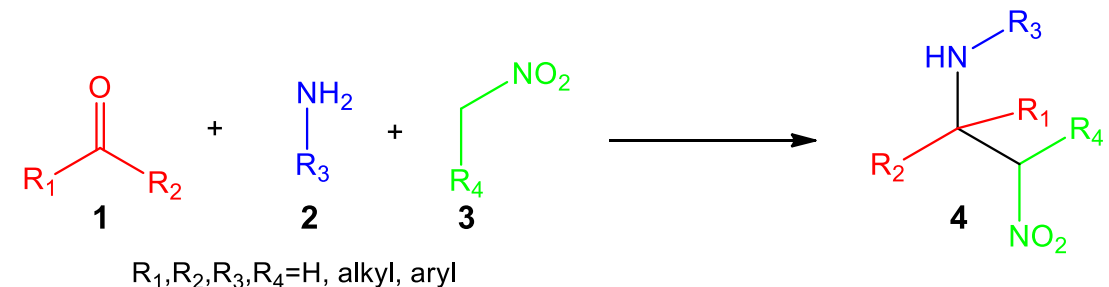




\section{Figure 3}

Catalyst screening tests carried out on the nitro-Mannich step for the synthesis of 6 using previously prepared $\mathrm{N}$-benzylideneaniline (5) and nitromethane (3a).

The highlighted row in green indicates the preferred catalyst used for subsequent optimisation tests.

$\mathrm{a}=$ All reactions were carried out at room temperature, in a 1:10 5:3a ratio at $2.5 \mathrm{mmol} . \mathrm{b}=$ Isolated yield. $\mathrm{c}=$ No product

\begin{tabular}{|c|c|c|c|c|c|c|c|}
\hline & & 5 & $3 a$ & & & & \\
\hline Entry $^{a}$ & Catalyst & $\begin{array}{l}\text { Time } \\
\text { (h) }\end{array}$ & $\begin{array}{l}\text { Yield } \quad 6 \\
(\%)^{b}\end{array}$ & Entry $^{\mathrm{a}}$ & Catalyst & $\begin{array}{l}\text { Time } \\
\text { (h) }\end{array}$ & $\begin{array}{l}\text { Yield } 6 \\
(\%)^{\mathrm{b}}\end{array}$ \\
\hline 1 & No & 168 & $-^{c}$ & 14 & KF-silica (53 wt $\%)$ & 168 & 39 \\
\hline 2 & $\mathrm{~K}_{2} \mathrm{CO}_{3}(20 \mathrm{~mol} \%)$ & 120 & 49 & 15 & KF-montmorillonite (14 wt $\%)$ & 168 & 51 \\
\hline 3 & $\mathrm{Na}_{2} \mathrm{CO}_{3}(10 \mathrm{~mol} \%)$ & 96 & 50 & 16 & KF/ basic alumina (33 wt\%) & 120 & 54 \\
\hline 4 & $\mathrm{Cs}_{2} \mathrm{CO}_{3}(200 \mathrm{~mol} \%)$ & 110 & 10 & 17 & CuI/Amberlyst A-21 (5 mol\%) & 48 & 75 \\
\hline 5 & $\mathrm{NaHCO}_{3}(10 \mathrm{~mol} \%)$ & 120 & 50 & 18 & $\mathrm{CuCl} / \mathrm{A}-21(5 \mathrm{~mol} \%)$ & 96 & 35 \\
\hline 6 & $\mathrm{KOH}(100 \mathrm{~mol} \%)$ & 120 & 49 & 19 & $\mathrm{AgNO}_{3} / \mathrm{A}-21(5 \mathrm{~mol} \%)$ & 168 & 18 \\
\hline 7 & Silica (332 mol\%) & 48 & 69 & 20 & CuI/Montmorillonite $\mathrm{K}-10$ (5 wt\%) & 168 & 21 \\
\hline 8 & Basic Alumina (400 mol\%) & 72 & 59 & 21 & $\mathrm{CuI} /$ basic Alumina $(3 \mathrm{~mol} \%)$ & 40 & 50 \\
\hline 9 & $\begin{array}{l}\text { Amberlyst A-21 (33mg/ } \\
1 \mathrm{mmol} \text { of aldimine) }\end{array}$ & 96 & 39 & 22 & $\begin{array}{l}\text { CuI-doped basic Alumina ( } 200 \\
\text { mol\% CuI and } 400 \text { mol\% alumina) }\end{array}$ & 40 & 22 \\
\hline 10 & $\begin{array}{l}\text { Amberlite }{ }^{\circledR} \quad \text { IRA-400 } \mathrm{Cl}^{-} \\
\left(0.5 \mathrm{~g} / 1.5 \mathrm{mmol} \text { of } \mathrm{Cl}^{-}\right)\end{array}$ & 168 & 50 & 23 & $\mathrm{CuI}(5 \mathrm{~mol} \%)$ & 120 & 23 \\
\hline 11 & Nafion SAC-13 (30 wt $\%)$ & 120 & 32 & 24 & $\begin{array}{l}\mathrm{Ag}(\mathrm{I}) / \text { Montmorillonite-K-10 (7 } \\
\mathrm{wt} \%)\end{array}$ & 168 & 36 \\
\hline 12 & $\begin{array}{l}\text { Montmorillonite K-10 (20 } \\
\text { wt } \%)\end{array}$ & 96 & 45 & 25 & PEG-400 (5 mL) & 120 & 29 \\
\hline 13 & $\mathrm{PPA} / \mathrm{SiO}_{2}(20 \mathrm{wt} \%)$ & 72 & $-^{\mathrm{c}}$ & & & & \\
\hline
\end{tabular}


Figure 4 (on next page)

One-pot sequential multicomponent nitro-Mannich reaction between benzaldehyde $1 a$, aniline $2 a$, and nitromethane $3 a$ to form $\beta$-nitroamine product 6 . 


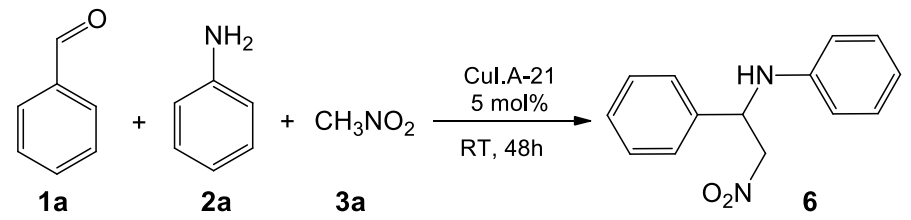


Figure $\mathbf{5}$ (on next page)

Optimisation trials on the model nitro-Mannich reaction between benzaldehyde (1a), aniline (2a) and nitromethane (3a). Highlighted cells indicate a change in factor. Cell highlighted in red indicates the entry with ideal conditions.

$a=$ Reaction was performed on a $2.5 \mathrm{mmol}$ scale. $b=$ Isolated product yield. $c=$ Original conditions in which copper(I) iodide was used during the screening of catalysts on the model reaction. $d=$ Trial was done using a batch of catalyst which was previously prepared and stored in a desiccator under vacuum with a loading of $1.35 \mathrm{mmol} \mathrm{Culg}^{-1} . \mathrm{e}=$ Trials were done using a newly prepared batch of catalyst with a loading of $1.52 \mathrm{mmol} \mathrm{Cul} \mathrm{g}^{-1}$ 


\begin{tabular}{|l|l|l|l|l|l|l|l|}
\hline Entry $^{\text {Aldehyde }}$ & $\begin{array}{l}\text { Amine }^{\mathrm{a}} \\
\mathbf{1 a}(\mathrm{mmol})\end{array}$ & $\begin{array}{l}\text { Nitroalkane }^{\mathrm{a}}(\mathrm{mmol}) \\
\mathbf{3 a}(\mathrm{mmol})\end{array}$ & $\begin{array}{l}\text { Catalyst } \\
(\mathrm{mol} \%)\end{array}$ & $\begin{array}{l}\text { Temperature } \\
\left({ }^{\circ} \mathrm{C}\right)\end{array}$ & $\begin{array}{l}\text { Time } \\
(\mathrm{h})\end{array}$ & $\begin{array}{l}\text { Yield }^{\mathrm{b}} \\
\mathbf{6}(\%)\end{array}$ \\
\hline $1^{\mathrm{c}, \mathrm{d}}$ & 1 & 1 & 10 & 5 & RT & 48 & 78 \\
\hline $2^{\mathrm{d}}$ & 1 & 1 & 10 & 20 & RT & 48 & 74 \\
\hline $3^{\mathrm{d}}$ & 1 & 1 & 10 & 10 & RT & 48 & 72 \\
\hline $4^{\mathrm{d}}$ & 1 & 1 & 10 & 2 & RT & 60 & 44 \\
\hline $5^{\mathrm{d}}$ & 1 & 1 & 10 & 1 & RT & 60 & 43 \\
\hline $6^{\mathrm{d}}$ & 1 & 1 & 7.5 & 5 & RT & 48 & 74 \\
\hline $7^{\mathrm{d}}$ & 1 & 1 & 5 & 5 & RT & 48 & 82 \\
\hline $8^{\mathrm{d}}$ & 1 & 1 & 2.5 & 5 & RT & 48 & 62 \\
\hline $9^{\mathrm{d}}$ & 1 & 1 & 1 & 5 & RT & 48 & 40 \\
\hline $10^{\mathrm{d}}$ & 1 & 1 & 5 & 5 & RT under $\mathrm{N}_{2}$ & 60 & 67 \\
\hline $11^{\mathrm{e}}$ & 1 & 1 & 5 & 5 & 100 & 2 & 86 \\
\hline $12^{\mathrm{e}}$ & 1 & 1 & 5 & 5 & 80 & 5 & 84 \\
\hline $13^{\mathrm{e}}$ & 1 & 1 & 5 & 5 & 60 & 10 & 80 \\
\hline $14^{\mathrm{e}}$ & 1 & 1 & 5 & 5 & RT & 48 & 81 \\
\hline $15^{\mathrm{e}}$ & 1 & 1 & 4 & 5 & 100 & 2 & 70 \\
\hline $16^{\mathrm{e}}$ & 1 & 1 & 3 & 5 & 100 & 2 & 62 \\
\hline
\end{tabular}




\section{Figure 6 (on next page)}

Effect of solvent on the multicomponent nitro-Mannich reaction between benzaldehyde $1 \mathrm{a}$, aniline $2 \mathrm{a}$ and nitromethane $3 \mathrm{a}$.

$a=$ Reaction was performed at $2.5 \mathrm{mmol}$ scale with a 5 -fold excess $3 \mathrm{a} . \mathrm{b}=5 \mathrm{~mL}$ solvent was added in each trial 


\begin{tabular}{|l|l|l|l|l|}
\hline Entry $^{\mathrm{a}}$ & Solvent $^{\mathrm{b}}$ & Temperature $\left({ }^{\circ} \mathrm{C}\right)$ & Time $(\mathrm{h})$ & Yield 6 (\%) \\
\hline 1 & - & 100 & 2 & 86 \\
\hline 2 & toluene & 100 & 2 & 28 \\
\hline 3 & chloroform & reflux & 2 & 20 \\
\hline 4 & ethyl acetate & reflux & 2 & 25 \\
\hline 5 & acetonitrile & reflux & 2 & 28 \\
\hline 6 & methanol & reflux & 2 & 84 \\
\hline 7 & water & 100 & 2 & 79 \\
\hline
\end{tabular}


Figure 7

Substrate scope with various aldehydes for the synthesis of $\beta$-nitroamines 7a-p.

$a=$ isolated pure yield, $b=$ No product formation 


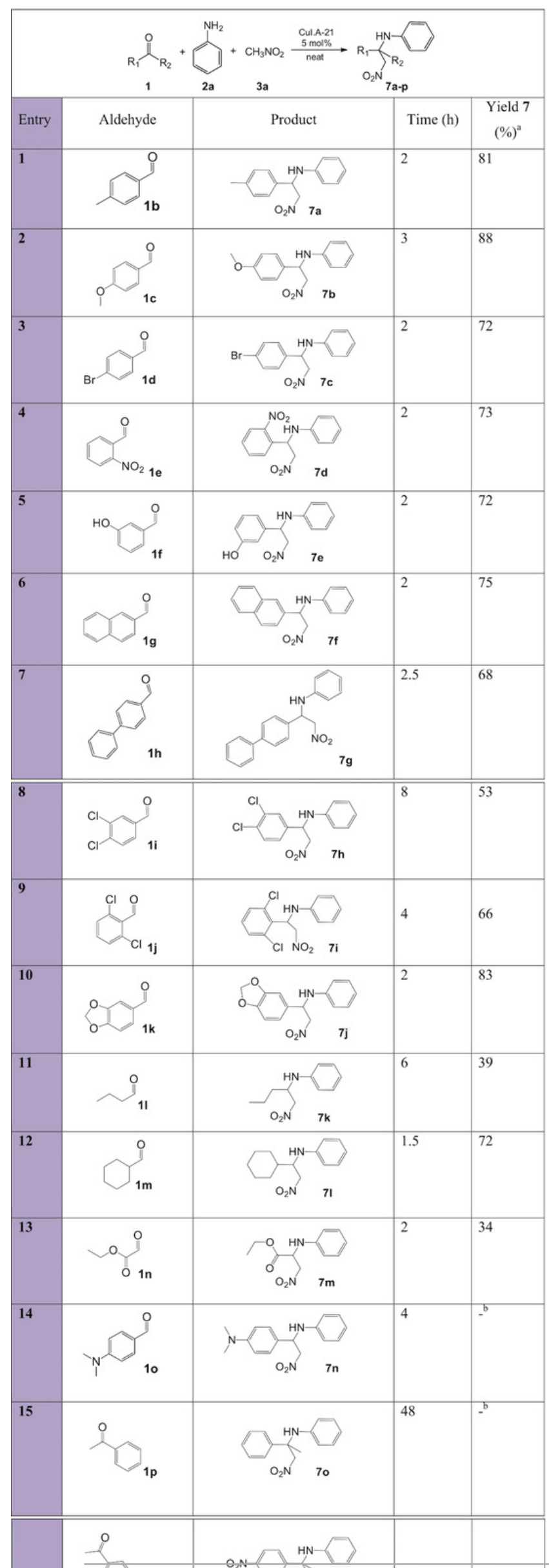


Figure 8

Substrate scope with various amines for synthesis of $\beta$-nitroamines 8a-n.

$a=$ Isolated pure yield. $b=$ No product formation 


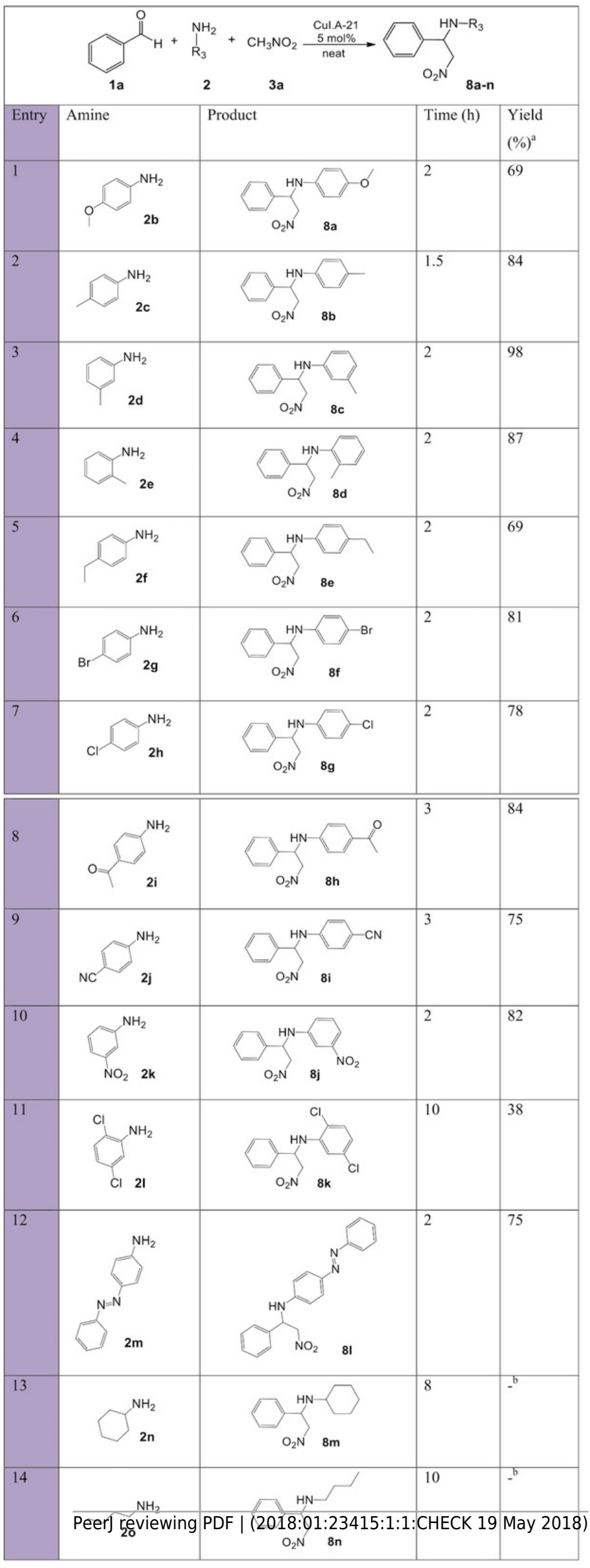


Figure 9

Substrate scope with various nitroalkanes for the synthesis of $\beta$-nitroamines 9a-b.

$a=$ Isolated pure yield. $b=$ Determined by ${ }^{1} \mathrm{H}$ NMR spectroscopy or from the isolated yield when separation between the two pairs of diastereomers was possible

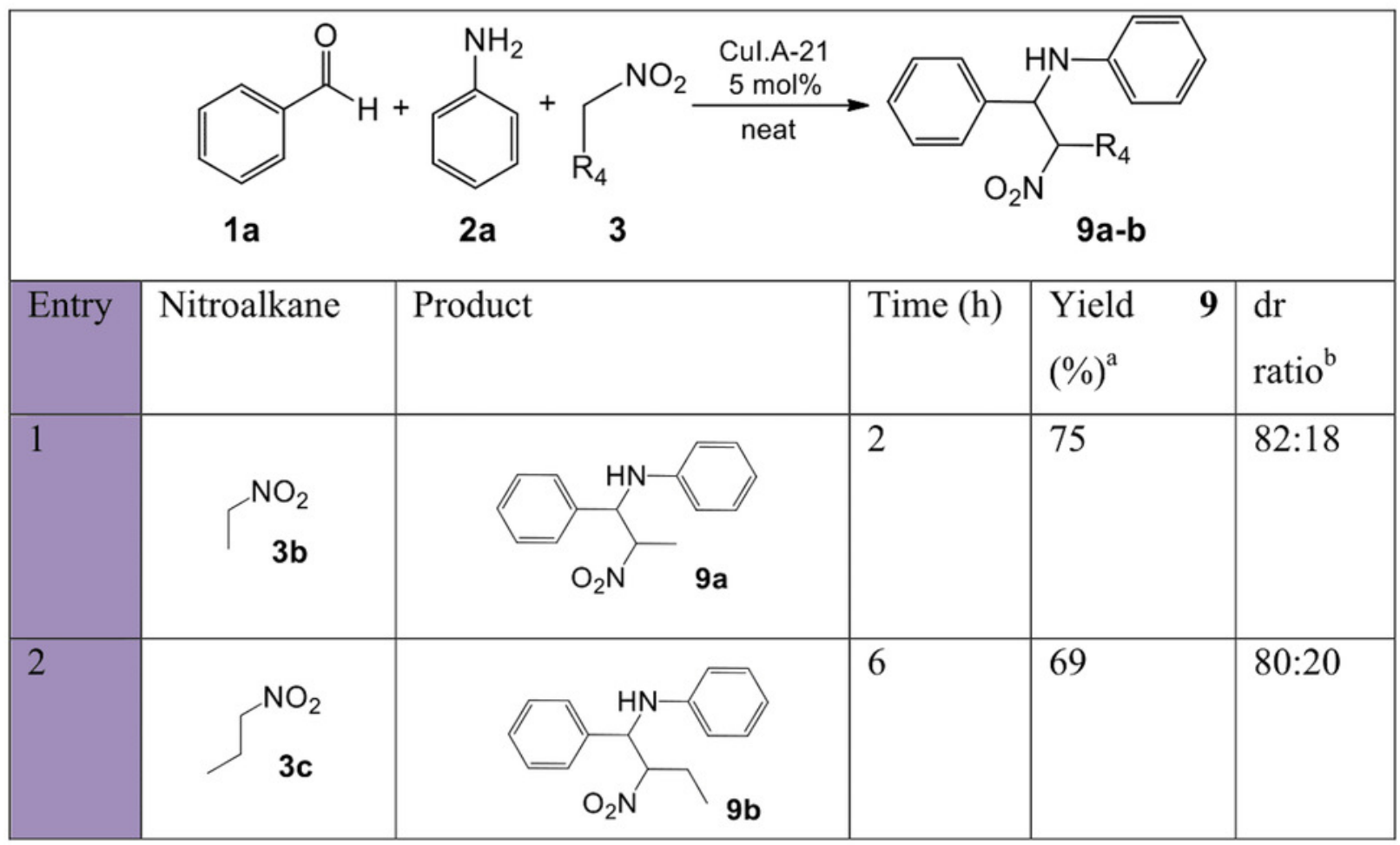




\section{Figure 10}

Substrate scope with mixed starting materials for the synthesis of $\beta$-nitroamines $4 a-1$.

$a=$ Isolated pure yield. $b=$ No product formation. $c=$ Determined by ${ }^{1} \mathrm{H}$ NMR spectroscopy $\cdot d=$ Not available 


\begin{tabular}{|c|c|c|c|c|c|c|c|}
\hline & & $\mathrm{R}_{1}^{\stackrel{O}{N}}$ & $\begin{array}{l}\prod_{\mathrm{R}_{3}}^{\mathrm{NH}_{2}} \\
\\
2\end{array}$ & $\int_{\mathrm{R}_{4}}^{\mathrm{NO}_{2}}$ & & & \\
\hline Entry & Aldehyde & Amine & Nitroalkane & Product & $\begin{array}{l}\text { Time } \\
\text { (h) }\end{array}$ & $\begin{array}{l}\text { Yield } 4 \\
(\%)^{\mathrm{a}}\end{array}$ & $\begin{array}{l}\mathrm{dr} \\
\text { ratio }^{\mathrm{c}}\end{array}$ \\
\hline 1 & $1 \mathrm{~b}$ & $2 k$ & $3 \mathbf{a}$ & & 2 & 75 & $-{ }^{d}$ \\
\hline 2 & $1 \mathrm{c}$ & $2 \mathrm{~h}$ & $3 \mathbf{a}$ & & 1.5 & 94 & $-d$ \\
\hline 3 & 1f & $2 b$ & $3 \mathbf{a}$ & & 2 & 56 & $-{ }^{d}$ \\
\hline 4 & $1 \mathrm{~m}$ & $2 \mathrm{~h}$ & $3 \mathbf{a}$ & & 2 & 67 & $-d$ \\
\hline 5 & $1 \mathrm{e}$ & $2 \mathrm{c}$ & $3 \mathbf{a}$ & & 2 & 77 & $-{ }^{d}$ \\
\hline 6 & 1e & $2 k$ & $3 a$ & & 12 & $-b$ & $-{ }^{d}$ \\
\hline 7 & 1d & $2 \mathrm{f}$ & $3 \mathbf{a}$ & & 3 & 67 & $-{ }^{d}$ \\
\hline 8 & 11 & $2 c$ & $3 \mathbf{a}$ & & 2 & 45 & $-{ }^{d}$ \\
\hline 9 & $1 \mathrm{~b}$ & $2 m$ & $3 \mathbf{a}$ & & 2 & 84 & $-{ }^{d}$ \\
\hline 10 & $1 \mathrm{c}$ & $2 \mathrm{i}$ & $3 \mathbf{b}$ & & 3 & 81 & $53: 47$ \\
\hline 11 & 1e & $2 \mathrm{c}$ & $3 \mathrm{c}$ & & 4 & 54 & $60: 40$ \\
\hline 12 & $\begin{array}{l}\mathbf{1 j} \\
\text { Peer] review }\end{array}$ & $\begin{array}{l}\text { 2d } \\
\text { g PDF | }\end{array}$ & 3c & $\therefore 1:$ CHEC & 8 & $-b$ & $-{ }^{d}$ \\
\hline
\end{tabular}


Figure 11(on next page)

A possible mechanism for the synthesis of $\beta$-nitroamine 12 by means of Cul.A-21 catalyst. 
(I)

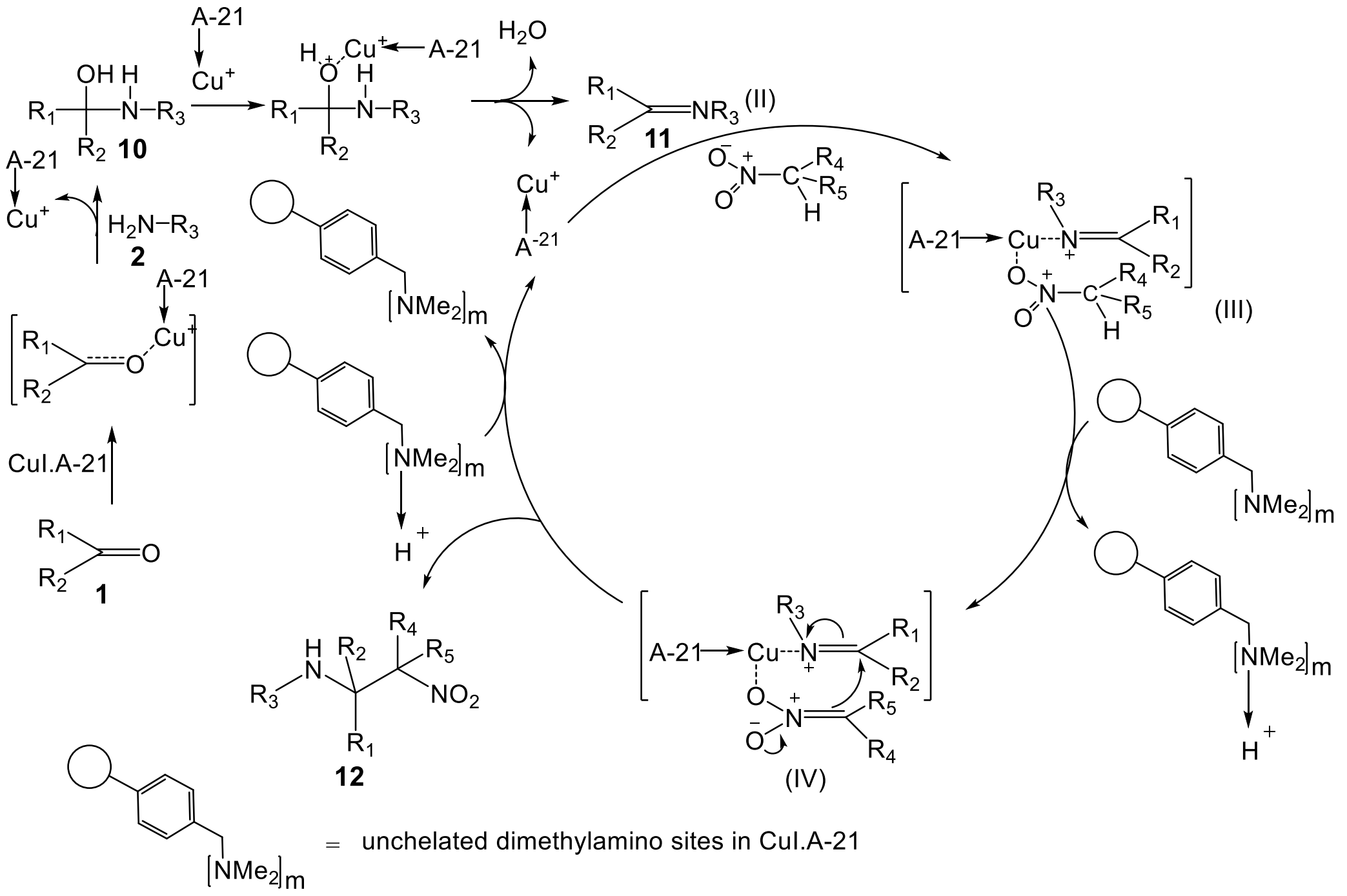


Figure 12 (on next page)

Comparison of the E-factor of unsupported Cul with Amberlyst supported copper(I) iodide for the synthesis of 6 .

$a=$ Based on the model reaction for the synthesis of $\mathbf{6}$ from $\mathbf{1 a}, \mathbf{2} \mathbf{a}$ and $\mathbf{3} \mathbf{a} . \mathbf{b}=$ =Isolated yield. 


\begin{tabular}{|c|c|c|}
\hline & Unsupported CuI & CuI.A-21 \\
\hline Reagent ratio $(\mathrm{mmol})^{\mathrm{a}}$ & \multicolumn{2}{|c|}{2.5 (aldehyde) : 2.5 (amine) : 12.5 (nitroalkane) } \\
\hline Catalyst Quantity & \multicolumn{2}{|c|}{$5 \mathrm{~mol} \%$} \\
\hline Yield $(\%)^{\mathbf{b}}$ & 28 & 86 \\
\hline Workup & Direct Loading & Catalyst Filtration \\
\hline Waste & $\begin{array}{l}0.125 \mathrm{mmol} \mathrm{CuI} \mathrm{catalyst} \\
10 \mathrm{mmol} \text { excess nitroalkane } \\
2.5 \mathrm{mmol} \mathrm{H}_{2} \mathrm{O} \text { waste }\end{array}$ & $\begin{array}{l}10 \mathrm{mmol} \text { excess nitroalkane } \\
2.5 \mathrm{mmol} \mathrm{H}_{2} \mathrm{O} \text { waste }\end{array}$ \\
\hline E-factor & $\begin{array}{lll}4.02 & \text { (including } & \text { catalyst } \\
\text { loss) } & & \end{array}$ & $\begin{array}{l}1.26 \text { (with complete catalyst } \\
\text { recovery) }\end{array}$ \\
\hline
\end{tabular}


Figure 13

Advantages of Amberlyst A-21 supported Cul as a heterogeneous catalyst. 


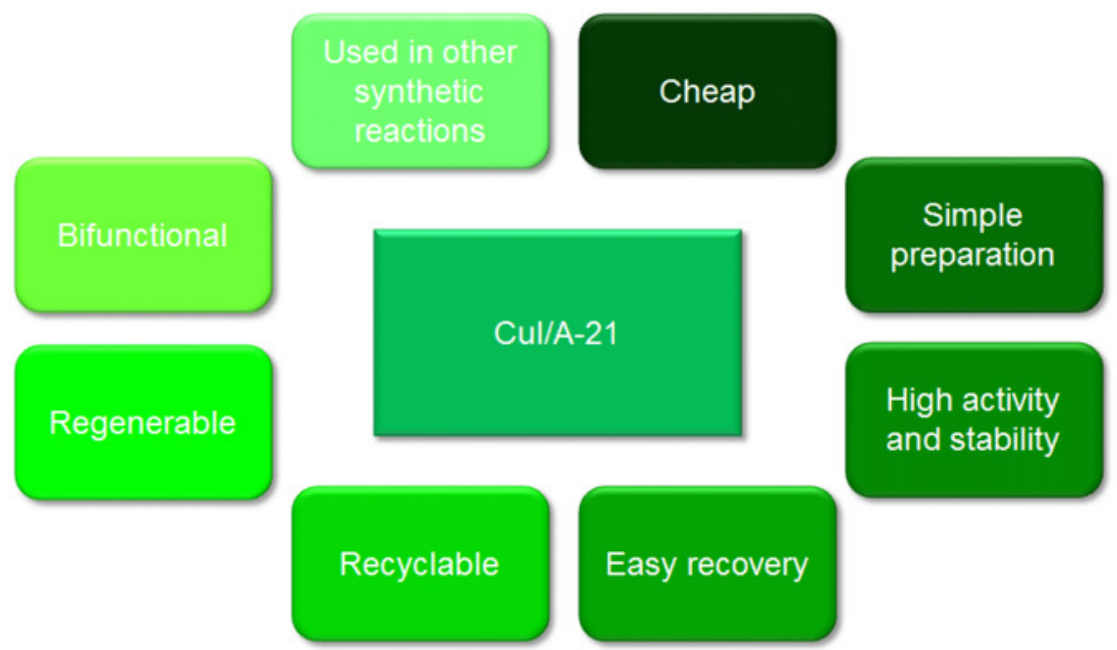


Figure 14(on next page)

Recycling test of Cul.A-21 for the synthesis of 6 in the model reaction. 


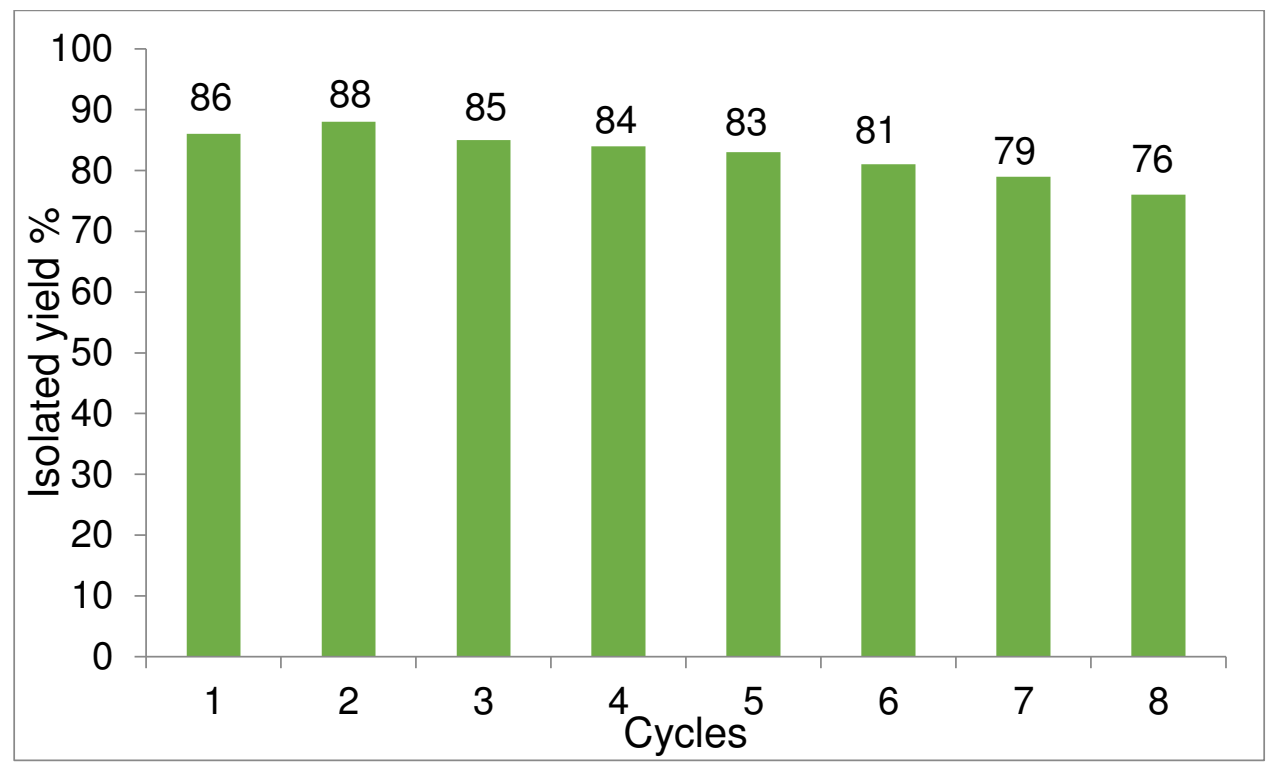


Figure 15(on next page)

Percentage of copper ions that leached into the solution from the heterogeneous catalyst Cul.A-21 throughout each cycle. 


\begin{tabular}{|l|l|}
\hline Cycle & $\% \mathrm{Cu}$ in solution \\
\hline 1 & 0.007 \\
\hline 2 & 0.36 \\
\hline 3 & 0.21 \\
\hline 4 & 0.095 \\
\hline 5 & 0.0002 \\
\hline 6 & 0.049 \\
\hline 7 & 0.15 \\
\hline 8 & 0.13 \\
\hline & Total $=1.00 \%$ \\
\hline
\end{tabular}

